\title{
Polymer-specific effects of bulk relaxation and stringlike correlated motion in the dynamics of a supercooled polymer melt
}

\author{
M. Aichele \\ Institut Charles Sadron, 6 rue Boussingault, 67083 Strasbourg, France and Institut für Physik, \\ Johannes Gutenberg-Universität, 55099 Mainz, Germany \\ Y. Gebremichael \\ Department of Chemical Engineering, University of Michigan, Ann Arbor, Michigan 48109 \\ and Chemical Physics Program, Institute for Physical Sciences and Technology, University of Maryland, \\ College Park, Maryland 20742 \\ F. W. Starr ${ }^{a)}$ \\ Center for Theoretical and Computational Materials Science and Polymers Division, National Institute \\ of Standards and Technology, Gaithersburg, Maryland 20899 \\ J. Baschnagel $\left.\right|^{\text {b) }}$ \\ Institut Charles Sadron, 6 rue Boussingault, 67083 Strasbourg, France \\ S. C. Glotzerc) \\ Departments of Chemical Engineering and Materials Science and Engineering, University of Michigan, \\ Ann Arbor, Michigan 48109
}

(Received 15 April 2003; accepted 11 June 2003; publisher error corrected 3 March 2004)

\begin{abstract}
We analyze dynamical heterogeneities in a simulated "bead-spring" model of a nonentangled, supercooled polymer melt. We explore the importance of chain connectivity on the spatially heterogeneous motion of the monomers. We find that when monomers move, they tend to follow each other in one-dimensional paths, forming strings as previously reported in atomic liquids and colloidal suspensions. The mean string length is largest at a time close to the peak time of the mean cluster size of mobile monomers. This maximum string length increases, roughly in an exponential fashion, on cooling toward the critical temperature $T_{\mathrm{MCT}}$ of the mode-coupling theory, but generally remains small, although large strings involving ten or more monomers are observed. An important contribution to this replacement comes from directly bonded neighbors in the chain. However, mobility is not concentrated along the backbone of the chains. Thus, a relaxation mechanism in which neighboring mobile monomers along the chain move predominantly along the backbone of the chains, seems unlikely for the system studied. () 2003 American Institute of Physics.
\end{abstract}

[DOI: $10.1063 / 1.1597473]$

\section{INTRODUCTION}

Many liquids supercooled below the melting temperature $T_{m}$ transform into a glass, a solid phase without long-range structural order, at the glass transition temperature $T_{g}$. The liquid well above $T_{g}$ and the amorphous solid below $T_{g}$ are structurally similar, yet their dynamics are very different. In the temperature interval $T_{g} \lesssim T \lesssim T_{m}$ the relaxation time slows dramatically on cooling, typically by more than ten orders of magnitude. ${ }^{1-3}$

A contributing factor for this dramatic change in the liquid dynamics may be attributed to the caging and the resulting emergence of spatially heterogeneous dynamics upon cooling toward $T_{g} \cdot{ }^{4-7}$ The term "dynamic heterogeneity" means that the amorphous packing in the supercooled state

\footnotetext{
a) Present address: Department of Physics, Wesleyan University, Middletown, CT 06459.

b) Author to whom correspondence should be addressed. Electronic mail: baschnag@ics.u-strasbg.fr

c) Author to whom correspondence should be addressed. Electronic mail: sglotzer@umich.edu
}

engenders aggregates ("subensembles") of particles with enhanced or reduced mobility relative to the average on intermediate time scales. These high and low mobility regions fluctuate throughout the sample with a finite lifetime.

To test this idea experimentally several techniques, such as multidimensional $\mathrm{NMR},{ }^{8,9}$ optical bleaching, ${ }^{10}$ nonresonant spectral hole burning ${ }^{11}$ or solvation dynamics, ${ }^{12}$ have been applied to a variety of glass formers (for reviews see Refs. 4-6). These experiments show that it is possible to select subensembles of slow or fast particles close to $T_{g}$. Although the possibility of detecting such subsets suggests that the fast or slow particles may be spatially correlated, direct evidence of this is difficult to extract by these experimental approaches. ${ }^{4,13}$

Information on this correlation has, however, become accessible in recent experiments on colloidal suspensions. ${ }^{14,15}$ Colloidal suspensions undergo a glass transition driven by density, ${ }^{16}$ which, for hard-sphere colloids, is well described by the idealized mode-coupling theory (MCT). ${ }^{17-20}$ Individual particle trajectories can be monitored by confocal spectroscopy. ${ }^{14,15}$ This new technique provides the same in- 
formation as computer simulations on atomic liquids. By scaling the time scales of the colloidal system and the simulated system by a common characteristic relaxation time (such as the $\alpha$-relaxation time), the dynamics of the two systems may be compared. The experiments of Ref. 14 revealed that the fastest particles form clusters whose size increases as the glass transition is approached. These findings closely agree with the results obtained from computer simulations of various model glass formers, ${ }^{7}$ such as binary soft-spheres ${ }^{21}$ and Lennard-Jones (LJ) mixtures, ${ }^{22-24}$ polymer melts, ${ }^{25}$ and water ${ }^{26}$ (see also Refs. 27-29 for related work on two- and three-dimensional hard spheres as well as Ref. 30 for dynamical heterogeneities in a LJ-mixture below $T_{g}$ ).

In this paper we analyze molecular dynamics simulations of a nonentangled polymer melt above its glass transition temperature. We study the commonly employed finitely extensible nonlinearelastic (FENE) bead-spring model of linear polymer chains, each containing $N=10$ monomers. Our results are based on molecular dynamics simulations of $102-120$ chains, depending on temperature. We study an isobaric path with pressure $p=1$ for 10 temperatures in the range $0.46 \leqslant T \leqslant 1.0$. These temperatures correspond to monomer densities ranging between $1.04 \geqslant \rho \geqslant 0.91$. The simulations were performed in the canonical ensemble using a Nosé-Hoover thermostat. Further simulation details can be found in Refs. 31-33. For reference, we quote the critical temperature of ideal mode coupling theory, $T_{\mathrm{MCT}}=0.450$ $\pm 0.005 .^{34-36}$

This model has been extensively investigated in the supercooled regime above $T_{\mathrm{MCT}} \cdot{ }^{34-38}$ In this temperature regime, the liquid exhibits dynamical heterogeneities. This was evidenced by two approaches. The first consisted in calculating spatial correlations between the displacements of different monomers via a "displacement-displacement" correlation function. ${ }^{31}$ Reference 31 showed that the strength of these correlations, as measured by a generalized susceptibility given by the volume integral of the displacementdisplacement correlation function, depends on time, is largest for times in the $\alpha$-relaxation regime, and grows on cooling toward $T_{\mathrm{MCT}}$. The susceptibility was found to follow a power law behavior, exhibiting an apparent divergence at $T_{\mathrm{MCT}}$. Moreover, the time scale corresponding to the maximum in the susceptibility increases with decreasing $T$ as a power law, following the same scaling with $T-T_{\mathrm{MCT}}$ as the diffusion coefficient, which is characterized by an exponent less than that of the $\alpha$-relaxation time.

These findings were supported by a second approach ${ }^{25}$ which identified clusters of highly mobile particles and analyzed their size distribution as a function of time and temperature close to $T_{\mathrm{MCT}}$. There it was demonstrated that the mobile monomers form clusters that are largest when measured over a characteristic time scale $t_{\mathrm{clu}}^{\max }$ in the late- $\beta /$ early- $\alpha$ regime. The time scale $t_{\text {clu }}^{\max }$ appears to diverge as a power law with $T-T_{\mathrm{MCT}}$, with an exponent smaller than that of the diffusion coefficient. Furthermore, it was found that the cluster size distribution approaches a power law as $T \rightarrow T_{\mathrm{MCT}}$ from above. ${ }^{25}$

The nature of dynamical heterogeneity studied in this system in terms of the displacement-displacement correlation function or the cluster analysis agrees closely with that in simple LJ mixtures. ${ }^{22-24}$ In the LJ system, the nature of cooperative motion was further investigated, and it was discovered that particles follow each other in quasi-onedimensional paths. This analysis has not been performed for a polymer system. In particular, it is interesting to ask whether, if strings exist, chain connectivity plays any role. Therefore the goals of this work are twofold: (i) To ascertain the tendency (or lack thereof) for monomers in the supercooled melt to follow each other in stringlike paths, and (ii) to study the influence of chain connectivity on dynamical heterogeneity and strings.

This paper addresses polymer-specific relaxation properties of polymer melts via detailed analysis of the motion of bonded and nonbonded monomers. To this end, we have considerably extended the simulations of Refs. 25 and 31 to longer times at the lowest and the highest temperatures, and we investigated spatial correlations in the motion of the highly mobile monomers, as in Refs. 22-24. The paper is organized as follows: Section II reviews the mean square displacement and non-Gaussian parameter for polymer chains and for monomers (chain segments). These quantities are useful reference points for the subsequent analysis. In Sec. III we present a detailed analysis of the dynamic properties and cooperative motion in the melt. In this section we demonstrate the occurrence of stringlike motion and investigate the influence of chain connectivity on this motion. A summary of the main results is given in Sec. IV.

\section{DYNAMICS OF THE MELT}

\section{A. Mean-square displacements}

The change in dynamics on cooling can be observed by examining the mean-square displacement (MSD) of a monomer $g_{0}(t)$ and of the center-of-mass of a chain $g_{3}(t)$

$g_{0}(t)=\left\langle\left[\mathbf{r}_{i}(t)-\mathbf{r}_{i}(0)\right]^{2}\right\rangle, \quad g_{3}(t)=\left\langle\left[\mathbf{R}_{c}(t)-\mathbf{R}_{c}(0)\right]^{2}\right\rangle$.

Here, $\langle\cdot\rangle$ denotes the canonical ensemble average, $\mathbf{r}_{i}(t)$ is the position of monomer $i$ at time $t$, and $\mathbf{R}_{c}(t)$ is the position of the center-of-mass of chain $c$ at time $t$. These quantities are shown in Fig. 1.

At short times, $g_{0}(t) \sim t^{2}$ for all temperatures (regime of ballistic motion). At $T=1$, we observe subdiffusive behavior for longer times, $g_{0} \sim t^{x}(x \simeq 0.65)$. This subdiffusive behavior can be attributed to chain connectivity which determines the monomer dynamics for $g_{0} \gtrsim 1 \quad(=$ one monomer diameter). ${ }^{34,38}$ If $g_{0}$ is comparable to the size of the chain, free diffusion sets in $\left(g_{0} \sim t\right)$.

At low temperature, this scenario is interrupted by an intermediate time window where $g_{0}$ increases slowly ("plateau regime"). The plateau reflects a temporary localization of the monomers by their nearest neighbors ("cage") because $g_{0}$ is of the order of $10 \%$ of the monomer diameter $\left(g_{0}\right.$ is close to $6 r_{0, c}^{2}$ with $r_{0, c} \simeq 0.095^{34,35}$ ). The intermediate time window encompassing the plateau and initial relaxation away from it is known as the " $\beta$-regime" in MCT. ${ }^{2}$ It precedes the final structural relaxation known as the " $\alpha$ - 


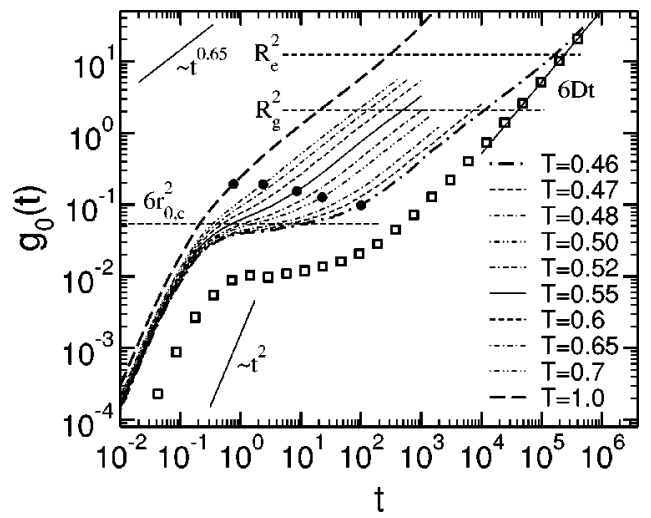

FIG. 1. Time-dependence of the MSD of all monomers, $g_{0}(t)$, for all $T$ studied and of the MSD of the center-of-mass, $g_{3}(t)$, at $T=0.46$ (shown by $\square)$ [see Eq. (1)]. Temperature decreases from the left $(T=1)$ to the right $(T=0.46)$ in the figure. The lowest temperature is slightly above $T_{\mathrm{MCT}}$ $\simeq 0.45$ (Refs. 34-36). The dashed horizontal lines indicate $6 r_{0, c}^{2}$ (Lindemann localization length $\left.r_{0, c} \simeq 0.095\right)$ and the values of the radius of gyration $R_{g}^{2}(=2.09)$ and of the end-to-end distance $R_{e}^{2}(=12.3)$. The filled circles $(\bullet)$ for $T=0.46,0.5,0.55,0.7,1$ mark the values of $g_{0}$ that correspond to the time $t_{\alpha_{2}}^{*}$ where the non-Gaussian parameter $\alpha_{2}$ is maximum (see Fig. 2). The solid line labeled $\sim t^{0.65}$ shows an effective power law describing the data in the regime $1 \leqq g_{0} \lesssim R_{e}^{2}$, where the connectivity between the monomers dominates the dynamics (Ref. 38). Two other solid lines indicate the behavior in the ballistic regime $\left(\sim t^{2}\right)$ and the diffusion of the center-ofmass $(6 D t)$.

regime." In the $\alpha$-regime a monomer begins to move subdiffusively due to the bonding to its neighbors and finally diffuses freely as soon as $g_{0}>R_{e}^{2}\left(R_{e}=\right.$ end-to-end distance).

Relative to $g_{0}$, the MSD of the center-of-mass is suppressed by about a factor of $1 / N$ in the $\beta$-regime. For longer times $g_{3}$ directly crosses over to free diffusion. There is no intervening subdiffusive regime because the center-of-mass is not subject to chain connectivity. These findings are in good agreement with recent theoretical predictions. ${ }^{39}$

\section{B. Non-Gaussian parameter}

In the limit $t \rightarrow 0$, the monomers move ballistically, and hence the self-part of the van Hove correlation function $G_{s}(\mathbf{r}, t)$, which is the probability for finding a particle at a distance $r$ at time $t$, is proportional to the MaxwellBoltzmann distribution, ${ }^{40}$ which has a Gaussian form. In the opposite limit $t \rightarrow \infty$, the polymers behave as if they were isolated Brownian particles subjected to a heat bath, and hence diffuse freely. Due to chain connectivity the monomers must follow the diffusive motion of the center-of-mass, and $G_{s}(\mathbf{r}, t)$ is again Gaussian.

At intermediate times, however, there may be deviations from Gaussian behavior. A possible means to measure these deviations is the non-Gaussian parameter $\alpha_{2}(t)^{40}$

$$
\alpha_{2}(t)=\frac{3\left\langle\left[\mathbf{r}_{i}(t)-\mathbf{r}_{i}(0)\right]^{4}\right\rangle}{5\left\langle\left[\mathbf{r}_{i}(t)-\mathbf{r}_{i}(0)\right]^{2}\right\rangle^{2}}-1,
$$

where $\mathbf{r}_{i}(t)$ is the position of monomer $i$ at time $t$. Similar to $\alpha_{2}(t)$, which quantifies deviations of the monomer dynamics from Gaussian behavior, one can also measure these deviations for the chain motion by calculating a non-Gaussian parameter $\alpha_{2}^{p}(t)$ for the polymers as

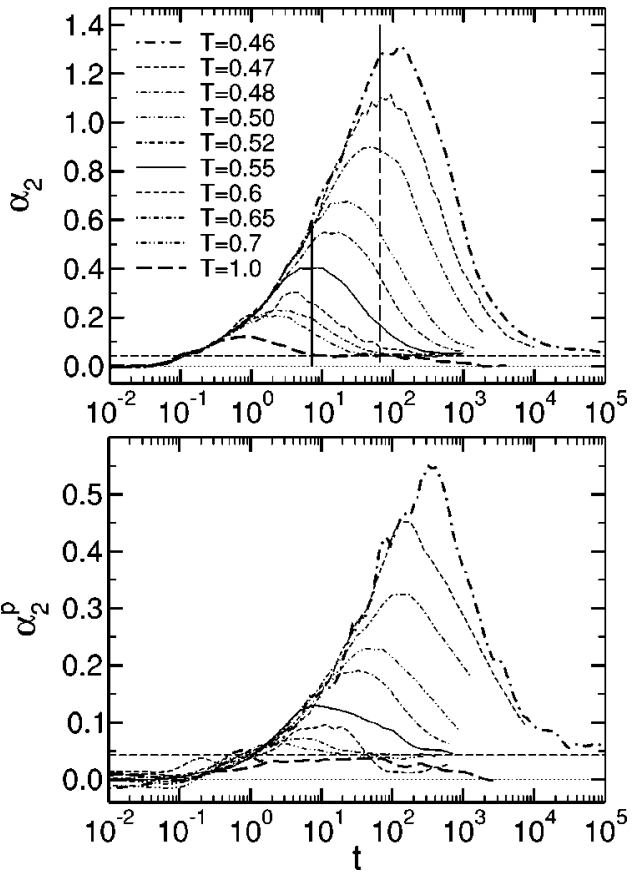

FIG. 2. Non-Gaussian parameter of the monomers $\alpha_{2}(t)$ (upper panel) and of the center-of-mass of the polymers $\alpha_{2}^{p}(t)$ (lower panel) vs time for different temperatures. $\alpha_{2}(t)$ and $\alpha_{2}^{p}(t)$ are defined in Eqs. (2) and (3). The temperature ranges from the high- $T$, normal liquid state above onset of caging ( $T=1$; thick dashed lines in both panels), to the supercooled state of the melt slightly above $T_{\mathrm{MCT}} \simeq 0.45$ ( $T=0.46$; thick dash-dotted lines). Temperature decreases from the bottom curve to the top curve in both panels. The dashed horizontal line $(=0.043)$ in the upper panel indicates a possible intermediate plateau toward which all $\alpha_{2}(t)$-curves could converge. This line is also included in the lower panel. The two vertical lines in the upper panel indicate the times $t_{\mathrm{clu}}^{\max }$ where clusters are maximum for $T=0.46$ at $t_{\text {clu }}^{\max }=65.85$ (dashed line) and for $T=0.55$ at $t_{\text {clu }}^{\max }=7.22$ (straight line).

$$
\alpha_{2}^{p}(t)=\frac{3\left\langle\left[\mathbf{R}_{c}(t)-\mathbf{R}_{c}(0)\right]^{4}\right\rangle}{5\left\langle\left[\mathbf{R}_{c}(t)-\mathbf{R}_{c}(0)\right]^{2}\right\rangle^{2}}-1,
$$

where $\mathbf{R}_{c}(t)$ is the position of the center-of-mass of chain $c$ at time $t$.

By definition, the non-Gaussian parameters vanish in the limits $t \rightarrow 0$ and $t \rightarrow \infty$. Otherwise, they are bounded from below because the mean-quartic displacement is always larger than or equal to the square of the mean-square displacement $\left[\alpha_{2}(t), \alpha_{2}^{p}(t) \geqslant-0.4\right]$. A negative value of the parameter means that the particles move on average less far than expected for a random walk, whereas a positive value implies that they move farther. The latter case is often observed in computer simulations ${ }^{41-46}$ and experiments ${ }^{14,15,47}$ on glass-forming liquids.

Figure 2 shows $\alpha_{2}(t)$ and $\alpha_{2}^{p}(t)$. With increasing time the amplitude of $\alpha_{2}(t)$ and $\alpha_{2}^{p}(t)$ increases to a maximum value which occurs at $t_{\alpha_{2}}^{*}$ for $\alpha_{2}(t)$ and at $t_{\alpha_{2}^{p}}^{*}$ for $\alpha_{2}^{p}(t)$. Notice that the peak height is larger for $\alpha_{2}(t)$ than for $\alpha_{2}^{p}(t)$ and that $t_{\alpha_{2}}^{*}$ and $t_{\alpha_{2}^{p}}^{*}$ are different. $t_{\alpha_{2}^{p}}^{*}$ is shifted by about half a decade to longer times for $T \leqslant 0.52$. Furthermore, a comparison of $t_{\alpha_{2}}^{*}$ and $t_{\mathrm{clu}}^{\max }$ (Ref. 25) indicates that both time scales occur in the late- $\beta$ /early- $\alpha$ relaxation regime, but that $t_{\text {clu }}^{\max }$ slightly precedes $t_{\alpha_{2}}^{*}$. There is a priori no reason why 
this particular non-Gaussian parameter and the mean cluster size should peak at the same time. However, with improved statistics, the previous estimates of $t_{\mathrm{clu}}^{\mathrm{max}}$ may shift closer to $t_{\alpha_{2}}^{*}$. In our subsequent analysis, we will mainly refer to $t_{\alpha_{2}}^{*}$ when comparing different time scales.

The small amplitude of $\alpha_{2}^{p}(t)$ may be attributed to the difference in packing of the monomers and of the chains. The monomers of our model exhibit an oscillatory pairdistribution function $g(r)^{35}$ whose shape and range are very similar to those found in simple dense liquids. In contrast to that, the pair-distribution function $g_{\mathrm{cm}}(r)$ for the centers-ofmass is fairly structureless, and resembles the $g(r)$ of a gas. ${ }^{48,49}$ This reflects the fact that polymers are soft, strongly interpenetrating objects and that the effective interaction between the centers-of-mass is weak. If this interaction were zero, there would be no resulting force on the center-ofmass. Then, the chain would diffuse freely (outside the ballistic regime). The small, nonzero $\alpha_{2}^{p}(t)$ at $T=1$ may thus be related to a weak force arising from the presence of other chains in the volume occupied by a polymer. ${ }^{50}$

On cooling the melt toward $T_{\text {MCT }}$ a pronounced maximum in time occurs for both non-Gaussian parameters. Since $g_{\mathrm{cm}}(r)$ is (nearly) temperature independent, ${ }^{48}$ the maximum of $\alpha_{2}^{p}$ cannot be attributed to enhanced interchain interactions at low $T$. The similarity between $\alpha_{2}$ and $\alpha_{2}^{p}$ rather suggests that the coupling between monomer and chain dynamics $^{39}$ drives the behavior of $\alpha_{2}^{p}(t)$. If the monomers of a chain are trapped in their cages and prevented from moving, the center-of-mass cannot move either. On the other hand, if a sufficient number of monomers move far during the time $t$, a large displacement of the center-of-mass results. As many monomers of the same chain are involved in this motion, a large displacement of the center-of-mass should take a longer time than for a single monomer. This explains why $t_{\alpha_{2}^{p}}^{*}$ is larger than $t_{\alpha_{2}}^{*}$.

In addition to the maximum, $\alpha_{2}$ shows two conspicuous features: First, there is a small, temperature independent step at $t \approx 0.1$. This time corresponds to the crossover of the monomer mean-square displacement (MSD) $g_{0}(t)$ from the ballistic to the plateau regime. The step can be more or less pronounced, depending on the microscopic properties of the system studied. ${ }^{42,45,46,51}$ Second, $\alpha_{2}(t)$ relaxes toward a plateau at long times before decaying to zero. This behavior is clearly visible for $T=1.0$, while lower $T$ are only indicative of a similar trend. Figure 2 suggests that the plateau value is the same for all $T$, but that the time when it is reached increases on cooling. For $T=1$ the plateau is attained when the MSD of all monomers is $\approx 1$, and this occurs at $t \approx 10$. For $T=0.46$, the plateau is only reached if $t \gtrsim 10^{5}$. This time corresponds to displacements of the order of the chain size for $T=0.46$, i.e., $R_{g}^{2}<g_{0}<R_{e}^{2}\left(R_{g}\right.$ and $R_{e}$ refer to the radius of gyration and the end-to-end distance of a polymer chain, respectively). Because the motion of the monomers becomes diffusive for $g_{0}>R_{e}^{2}$, one can speculate that the length of the plateau decreases with decreasing $T$.

For all $T$ the plateau occurs if $g_{0} \gtrsim 1$. This corresponds to times where the Rouse model ${ }^{52}$ is believed to describe the dynamics of nonentangled chains in the melt. ${ }^{53}$ In this model the displacements of the monomers and of the chains follow a Gaussian distribution, in which case, $\alpha_{2}(t)$ and $\alpha_{2}^{p}(t)$ should vanish at all times. The finite value of the plateau points to small, but systematic deviations from Rouse behavior. This value is approximately the same for both $\alpha_{2}$ and $\alpha_{2}^{p}$, and roughly agrees with the maximum of $\alpha_{2}^{p}$ found at $T=1$. The latter observation could imply that the occurrence of the plateau is related to the weak interactions between the centers of mass alluded to above.

In summary, the interpretation of Fig. 2 suggests that deviations from Gaussian behavior in our model might have two origins: The weak, temperature independent interaction between the centers-of-mass leads to small deviations in the long-time subdiffusive regime. Preceding the subdiffusive regime strong, $T$-dependent deviations occur due to the caging and subsequent correlated motion of monomers as observed in dense simple atomic liquids and colloids. This drives the sluggish glasslike relaxation of the monomer and, as a consequence, also that of the center-of-mass. Our subsequent analysis will focus on this correlated nature of the monomer dynamics.

\section{INVESTIGATION OF SPATIALLY CORRELATED MOTION}

\section{A. Definition of mobility}

We investigate the nature of the spatial correlations in monomer displacements as in Refs. 25 and 31 by focusing on the motion of the most mobile monomers in a given time. Following Ref. 25, we identify highly mobile monomers as those $6.5 \%$ of monomers with the largest scalar displacement at $t_{\mu}$. In this definition, the mobility of a monomer at any time $t_{\mu}$ is characterized by calculating the magnitude of its displacement within $t_{\mu}$ :

$$
\mu_{i}\left(t_{\mu}\right)=\left|\mathbf{r}_{i}\left(t_{\mu}\right)-\mathbf{r}_{i}(0)\right| \text {. }
$$

Other similar choices have been discussed elsewhere, ${ }^{24}$ but these alternate choices of mobility do not qualitatively affect our results.

\section{B. Mean-square displacement of mobile monomers}

We first characterize the motion of the mobile monomer subset, and investigate how that motion is affected by the choice of $t_{\mu}$. To this end, we calculate a specially defined MSD $g_{0, m}\left(t_{\mu}, t\right)$, which calculates the MSD, as a function of time, of a subset of monomers that are identified to be highly mobile in a specific time window $t_{\mu}$,

$$
g_{0, m}\left(t_{\mu}, t\right)=\frac{1}{0.065 V}\left\langle\sum_{i \in \mathcal{M}_{m}\left(t_{\mu}\right)}\left[\mathbf{r}_{i}(t)-\mathbf{r}_{i}(0)\right]^{2}\right\rangle .
$$

Here, $\mathcal{M}_{m}\left(t_{\mu}\right)$ denotes the subset of mobile monomers at time $t_{\mu}$ in the melt comprised of $M$ monomers. For all times the number of elements in $\mathcal{M}_{m}\left(t_{\mu}\right), 0.065 \mathrm{~V}$, remains fixed, although the composition of the subset changes with $t_{\mu}$; at different times different monomers will be among the $6.5 \%$ with the largest displacement. By fixing $t_{\mu}$ and varying $t$ we fix this composition for all $t$ and follow the motion of only those monomers that have been found to be mobile at $t_{\mu}$. However, we can also calculate the MSD of the mobile 

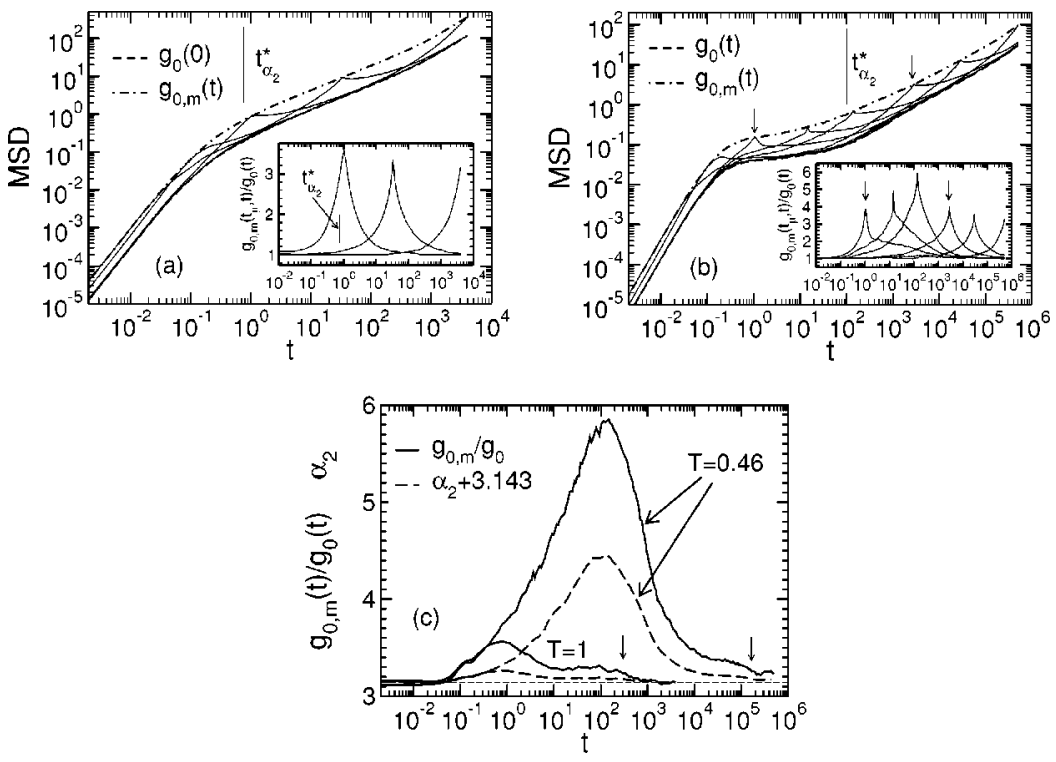

FIG. 3. Comparison of various MSD's at $T=1$ (a) and $T=0.46$ (b). The thick dashed curve is the average MSD, $g_{0}(t)$, of all monomers in the melt, whereas the dash-dotted curve represents the MSD of all mobile monomers, $g_{0, m}(t) . g_{0, m}(t)$ results from the concatenation of the cusps of the thin solid curves. Each of these solid curves shows the time evolution of $g_{0, m}\left(t_{\mu}, t\right)$, which is obtained by averaging over all those monomers that have been found to be mobile at $t=t_{\mu}$ (=time when the cusp occurs). The vertical solid lines in both panels show the time $t_{\alpha_{2}}^{*}$ where the non-Gaussian parameter $\alpha_{2}$ is maximum. In (b) the vertical arrows indicate two times $t_{\mu}$, one at the beginning of the plateau $\left(t_{\mu}=1.038\right)$ and in the $\alpha$-regime $\left(t_{\mu}=2634\right)$. The insets in (a) and (b) illustrate the difference between the average MSD and that of the mobile particles by showing $g_{0, m}\left(t_{\mu}, t\right) / g_{0}(t)$ for $t_{\mu}$ well beyond the ballistic regime. (c) compares $g_{0, m}(t) / g_{0}(t)$ to $\alpha_{2}(t)$. The non-Gaussian parameter is shifted upward by 3.143 [dashed horizontal line, see Eq. (6)]. The vertical arrows indicate the time when $g_{0}=R_{e}^{2}$ for $T=1$ and $T=0.46$, respectively.

monomers $g_{0, m}(t)$, as done in Ref. 25; this corresponds to the choice $t_{\mu}=t$ in Eq. (5). In that case, the composition of the subset may vary for different times.

Figures 3(a) and 3(b) compare $g_{0, m}(t)$ with $g_{0}(t)$ at $T$ $=1$ and $T=0.46$. Qualitatively, $g_{0}(t)$ and $g_{0, m}(t)$ exhibit the same behavior at both temperatures. However, there are quantitative differences. As expected, the mobile particles always move significantly farther than the average. At early and very late times, the ratio $g_{0, m}(t) / g_{0}(t)$ is approximately 3.14 [Fig. 3(c)]. This value can be predicted from the Gaussian behavior of the displacements in the ballistic and diffusive regimes. If $G_{s}(\mathbf{r}, t)$ is a Gaussian, $g_{0, m}(t) / g_{0}(t)$ is given by $^{54}$

$$
\frac{g_{0, m}(t)}{g_{0}(t)}=1+\frac{4}{0.065 \sqrt{9 \pi}}\left(x^{*}\right)^{3 / 2} e^{-x^{*}},
$$

where $x^{*}$ is determined by the normalization condition $(4 / \sqrt{\pi}) \int_{x^{*}}^{\infty} \mathrm{d} x x^{2} \exp \left(-x^{2}\right)=0.065$. This yields $x^{*} \simeq 3.6136$ so that $g_{0, m}(t) / g_{0}(t) \simeq 3.143$.

At intermediate times, $g_{0, m}(t) / g_{0}(t)$ is larger than 3.14 and behaves qualitatively in the same way as $\alpha_{2}$ [Fig. $3(\mathrm{c})] .{ }^{55}$ In particular, $g_{0, m}(t) / g_{0}(t)$ exhibits a maximum at $t$ $=t_{\alpha_{2}}^{*}$, the amplitude of which increases with decreasing $T$.

This illustrates again that the most mobile monomers move farther than expected for a random walk. The difference in mobility between all and the fastest monomers grows on cooling and is most pronounced in the late- $\beta /$ early- $\alpha$ regime.

Figures 3(a) and 3(b) also show $g_{0, m}\left(t_{\mu}, t\right)$, which illustrates how the monomers that are mobile at $t_{\mu}$ move at other times. As found in other simulated liquids, ${ }^{24}$ the mobile monomers do not belong to a special class of particles that are always faster than the average, since the system is ergodic. Rather they behave as the average for $t \ll t_{\mu}$, accelerate as $t$ approaches $t_{\mu}$, and finally relax back to the average for $t \gg t_{\mu}$. This cycle is symmetric on a log-scale at $T=1$ if $t_{\mu} \gtrsim t_{\alpha_{2}}^{*}$, i.e., in the subdiffusive regime [inset of Fig. 3(a)]. Similar behavior is found at $T=0.46$ for times beyond the plateau in the subdiffusive regime [inset of Fig. 3(b)]. However, the cycle is fairly asymmetric in the $\beta$-regime. If $t_{\mu}$ is at the beginning of the plateau [curve indicated by an arrow at $t_{\mu} \approx 1$ in Fig. 3(b)], the monomer accelerates fast, but takes a long time to relax back to $g_{0}(t)$, whereas the behavior is opposite if $t_{\mu}$ is in the $\alpha$-relaxation regime [curve indicated by an arrow at $t_{\mu} \approx 2600$ in Fig. 3(b)].

\section{Mobile monomers and the role of chain connectivity}

The previous subsection discussed the motion of mobile monomers without distinguishing whether or not they are connected to each other. The interplay of connectivity and mobility is one of the main issues we wish to address. Intuitively, one may expect that the bonds in a chain provide a preferred direction along which mobility can be "transmitted." To investigate this polymer-specific effect we calculate the mean contiguous segment length $N_{c, m}(t)$, which is defined as the average number of mobile monomers that are consecutively bonded to each other on a given polymer chain, averaged over all chains that contain at least one mobile monomer. Figure 4 illustrates this definition and shows that there can be multiple contiguous segments on a single chain. 


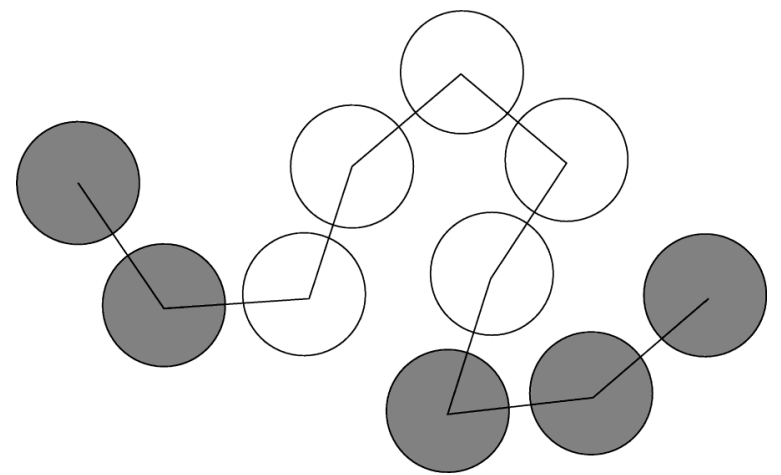

FIG. 4. A schematic diagram showing how the average length of contiguous segments of mobile monomers, $N_{c, m}\left(t_{\mu}\right)$, is defined. Assume that, at time $t_{\mu}$, only the shaded monomers are considered to be mobile. The first two monomers constitute a contiguous segment of length 2 , then there are five nonmobile monomers, followed by another contiguous segment of length 3 . So, the average length of contiguous mobile segments is $N_{c, m}\left(t_{\mu}\right)=2.5$.

\section{Correlations of mobile monomers in a chain}

Figure 5 shows $N_{c, m}(t)$ for all temperatures studied. In the ballistic regime we expect no correlations, ${ }^{25,31}$ and find $N_{c, m} \approx 1.06$. The value $N_{c, m} \approx 1.06$ could also be obtained by calculating $N_{c, m}$ after selecting $6.5 \%$ of monomers at random and labeling them as "mobile." Therefore, no significant dynamic correlations between bonded nearest neighbors exist in the ballistic regime.

Beyond the ballistic regime $N_{c, m}(t)$ increases, but never exceeds $\sim 1.5$ as long as $t \lesssim t_{\alpha_{2}}^{*}$. Thus, in the studied temperature interval, the relaxation mechanism does not correspond to the sliding motion of many consecutive monomers along the backbone of the chain, since that would require $N_{c, m}$ to be of order $N$. The small value of $N_{c, m}$ rather suggests that the relaxation in the $\beta$-regime is predominantly determined by the dense local packing of the melt and not by chain connectivity. This is consistent with the degree to which the ideal MCT for simple liquids is successful at describing the dynamics of polymer melts. However, this does not imply that chain connectivity is completely irrelevant. For $T \leqslant 0.7$ (below the onset of caging), $N_{c, m}(t)$ exhibits a maximum at $t_{\mathrm{seg}}^{\max }\left(z t_{\alpha_{2}}^{*}\right)$ in the time window of the late- $\beta /$ early- $\alpha$ process. The maximum increases upon cooling toward $T_{\mathrm{MCT}}$. Thus, the colder the melt, the larger the tendency for the mobile monomers to be nearest neighbors in the chain.

For times larger than $t_{\text {seg }}^{\max }$ spatial correlations between mobile monomers diminish. The length of the contiguous segments relaxes back to a minimum. The minimum occurs at $t_{\mathrm{seg}}^{\mathrm{min}}$ which roughly corresponds to the time where $g_{0}=1$ (subdiffusive regime). For $t>t_{\mathrm{seg}}^{\mathrm{min}}$, the crossover to free diffusion takes place and $N_{c, m}$ continuously increases, possibly converging to its upper limit $N$, because the displacement of the center-of-mass is predicated upon a concomitant motion of many monomers in the chain.

The occurrence of the maximum and the minimum suggests that there are two relaxation mechanisms at low temperature: One, occuring when $N_{c, m}(t)$ is a maximum, corresponds to the cage-breaking process. Here, clustering of highly mobile particles is most pronounced, irrespective of

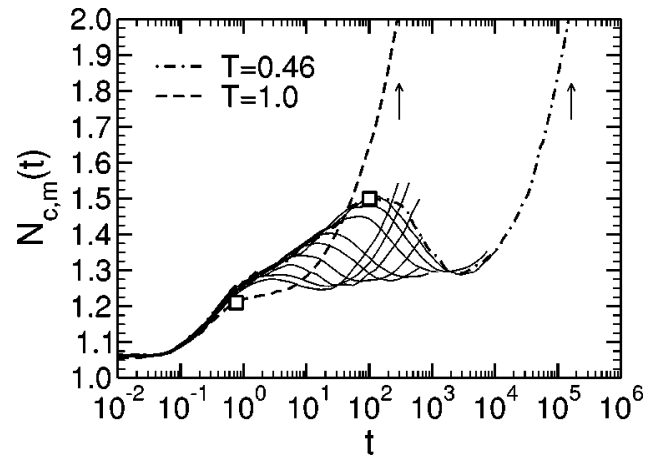

FIG. 5. Mean contiguous segment length $N_{c, m}(t)$ vs time for all temperatures. Besides $T=1$ (dashed curve) and $T=0.46$ (dash-dotted curve) the following temperatures are shown (solid curves from left to right): $T$ $=0.7,0.65,0.6,0.55,0.52,0.5,0.48,0.47$. For $T=1$ and $T=0.46$, the open squares indicate $t_{\alpha_{2}}^{*}$ and the arrows indicate the time when $g_{0}=R_{e}^{2}$.

whether they are bonded to each other or not. ${ }^{25}$ Cluster formation related to cage breaking is also observed in a binary LJ-mixture close to $T_{\mathrm{MCT}}{ }^{24}$ and in experiments on colloidal suspensions close to the glass transition. ${ }^{14}$ Thus, chain connectivity is not necessary for clustering. As in nonpolymeric liquids, the clustering is rather a consequence of the selfgenerated cooperativity between the local motion of the caged monomers in the cold melt. To a large extent, this cooperativity is lost as $N_{c, m}$ crosses over to the minimum. The minimum and the subsequent steep rise, which correspond to the second relaxation mechanism, are a signature of Rouse-type, polymer-specific dynamics because they are, at least as precursors, already present at $T=1$ where no caging occurs.

\section{Mobile end monomers}

In addition to $N_{c, m}$ we also analyzed the mobility of end monomers as compared to central monomers in polymer chains by calculating the fraction $f_{e, m}$ of mobile monomers that are end monomers. Figure 6(a) shows the time evolution of $N f_{e, m} / 2$ for all temperatures. The factor $N / 2$ takes into account that the a priori probability of finding an end monomer among the $N$ monomers of a chain is $2 / N$. If the mobility of the ends cannot be distinguished from the average, $N f_{e, m} / 2$ should be 1 . This is the case in the ballistic regime, where the monomers are independent of each other, and in the diffusive regime, where they follow the motion of the center-of-mass. At intermediate times, however, we find $N f_{e, m} / 2>1$, and hence chain ends are more mobile than inner monomers.

We compare $N f_{e, m} / 2$ with the ratio $g_{e}(t) / g_{0}(t)$, where $g_{e}(t)$ is the MSD of the end monomers [Fig. 6(b)]. The time dependence of $N f_{e, m} / 2$ closely agrees with that of $g_{e}(t) / g_{0}(t)$. This implies that the qualitative relationship between the motion of the mobile ends and that of all mobile monomers is not different from the average behavior of the melt. However, there are quantitative differences. For times outside the ballistic and diffusive regimes $N f_{e, m}(t) / 2$ is larger than $g_{e}(t) / g_{0}(t)$, except in the window of the intermediate plateau (MCT $\beta$-process), where $N f_{e, m}(t) / 2$ $\approx g_{e}(t) / g_{0}(t)$. 

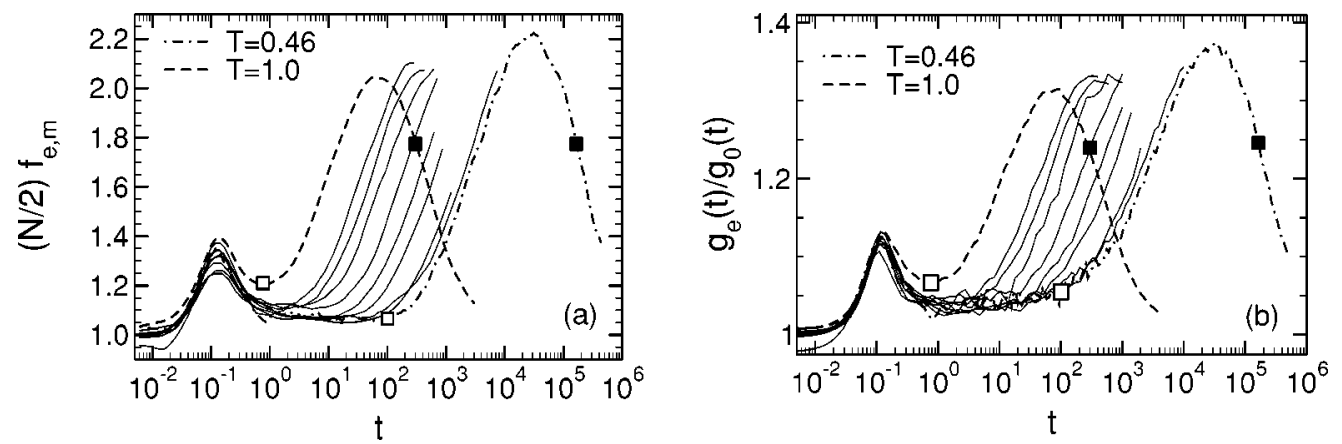

FIG. 6. (a) Fraction of mobile end monomers $f_{e, m}$ vs $t . f_{e, m}$ is multiplied by $N / 2(=5)$ to account for the fact that there are only two ends per chain. Besides $T=1$ (dashed curve) and $T=0.46$ (dash-dotted curve) the following temperatures are shown (solid curves from left to right): $T=0.7,0.65,0.6,0.55,0.52,0.5$, $0.48,0.47$. For $T=1$ and $T=0.46$, the open squares indicate $t_{\alpha_{2}}^{*}$, the filled squares the time when $g_{0}=R_{e}^{2}$. (b) Same as in (a), but for $g_{e}(t) / g_{0}(t) . g_{e}(t)$ is the MSD of the end monomers.

When leaving the ballistic regime $g_{e} / g_{0}$ first increases. This increase can be understood by the short-time expansion of the MSD of monomer $i^{40}$

$$
\begin{aligned}
\left\langle\left[\mathbf{r}_{i}(t)-\mathbf{r}_{i}(0)\right]^{2}\right\rangle & =2 t \int_{0}^{t} \mathrm{~d} t^{\prime}\left(1-\frac{t^{\prime}}{t}\right)\left\langle\mathbf{v}_{i}(t) \cdot \mathbf{v}_{i}(0)\right\rangle \\
& \approx 3 T t^{2}\left[1-\frac{\left\langle\left|\mathbf{F}_{i}\right|^{2}\right\rangle}{36 T} t^{2}\right](t \text { small }),
\end{aligned}
$$

where $\left\langle\mathbf{v}_{i}(t) \cdot \mathbf{v}_{i}(0)\right\rangle$ is the velocity auto-correlation function and $\mathbf{F}_{i}$ the total force on monomer $i$. Since an end is only bonded to one monomer, $\mathbf{F}_{i}$ is smaller than for inner monomers. Thus, one expects $g_{e} / g_{0}>1$ (and also $N f_{e, m} / 2>1$ ) for times just outside the ballistic regime. In our model the ratio continues to increase up to a maximum that occurs around $t \approx 0.13$. This is close to the time where the velocity autocorrelation function becomes negative. ${ }^{32}$ The inversion of the initial direction of the velocity is caused by rebounding collisions between a monomer and its neighbors. It is typical of dense liquids and must occur in the same way for end and inner monomers. Therefore, the difference in mobility should diminish and $g_{e} / g_{0}$ should decrease. In fact, the simulation shows that, for longer times, $g_{e} / g_{0}$ first decreases toward a minimum and then, at about $t \approx t_{\alpha_{2}}^{*}$, crosses over to a steep rise. The rise reaches a maximum close to the time where the MSD of the center-of-mass equals $R_{g}^{2}$. This roughly corresponds to the Rouse time $\tau_{\mathrm{R}}^{52}$ of our model. Thereafter, the transition to free diffusion takes place.

The enhanced mobility of the end monomers for $t>t_{\alpha_{2}}^{*}$ is not unexpected. The Rouse theory predicts $g_{e} / g_{0}=2$ in the time regime where the monomer displacement follows a $t^{1 / 2}$ behavior (i.e., for $t \leqslant \tau_{\mathrm{R}}$ ) ${ }^{52} \mathrm{In}$ the present simulation, the maximum of $g_{e} / g_{0}$ is smaller than 2, partially due to short chain effects. Longer chains may attain the Rouse prediction more closely if entanglements can be neglected. ${ }^{39}$

The double-peak structure of $g_{e} / g_{0}$ is present at all temperatures. When cooling the melt toward $T_{\mathrm{MCT}}$ two additional features can be observed: First, the second peak $(\alpha-$ relaxation) strongly shifts to longer times. This is the signature of the slowing down of structural relaxation. Second, the curves collapse in the time window of the minimum which deepens and evolves into a protracted plateau with decreasing $T$. Previous analysis ${ }^{34,35,37,38}$ showed that this time window corresponds to the MCT $\beta$-process where one expects temporary intermittence of particle motion due to the cage effect. ${ }^{2,56}$ In the $\beta$-regime, $g_{n}(t)$ is close to the localization length $6 r_{n, c}^{2},{ }^{2,39,56}$

$$
g_{n}(t)=6 r_{n, c}^{2}-6 h_{n} G(t) \quad(n=0, e) .
$$

Here, only the $\beta$-correlator $G(t)$ depends on time and temperature, whereas the other parameters $r_{n, c}$ and $h_{n}$ are independent of $T$ (close to $T_{\mathrm{MCT}}$ ). Since $6 h_{n} G(t)$ represents a small correction to $6 r_{n, c}^{2}$, Eq. (7) suggests $g_{e} / g_{0}$ $\approx\left(r_{e, c} / r_{0, c}\right)^{2} \quad(\simeq 1.046)$ in the time window of the $\beta$-relaxation. Figure 6 shows that $g_{e} / g_{0}$ and $N f_{e, m} / 2$ are indeed close to 1 in this time window. This illustrates that the different bonding of end and inner monomers does not crucially alter the dynamics in the $\beta$-regime. Here, the relaxation is determined by the local packing of a monomer and its nearest neighbors, which is (almost) the same for end and inner monomers (in our model).

\section{Stringlike motion}

The cluster analysis of Ref. 25 showed that the results for the spatial correlations between highly mobile monomers closely resemble those found in a binary Lennard-Jones mixture. ${ }^{24}$ References 23 and 24 also reveal that, in binary LJ liquids, the clusters are composed of smaller objects called "strings," which are sets of highly mobile particles that move in quasi-one-dimensional paths, replacing one another as they move. Following Ref. 24, any two mobile monomers $i$ and $j$ are considered to be in the same string if

$$
\min \left[\left|\mathbf{r}_{i}(t)-\mathbf{r}_{j}(0)\right|,\left|\mathbf{r}_{j}(t)-\mathbf{r}_{i}(0)\right|\right]<\delta .
$$

This equation means that monomer $i$ moved from $\mathbf{r}_{i}(0)$ to $\mathbf{r}_{i}(t)$ in time $t$, while the other monomer $j$ simultaneously approached the initial position of $i$ within a sphere of radius $\delta$. $\delta$ must be sufficiently smaller than the Lennard-Jones diameter $\sigma(=1)$ to guarantee that $j$ unambiguously replaces $i$. For the binary LJ-mixture, a good choice was $\delta=0.6{ }^{23}$

For the polymer model under consideration we find that Eq. (8) with $\delta=0.6$ may, in a very small percentage of cases, lead to ambiguities. With this choice of $\delta$, more than one monomer $j$ can "replace" $i$ simultaneously. If this happens 
frequently, a "string" may contain many Y-like portions, i.e., two monomers $j$ are equally likely to occupy at time $t$ the initial position of $i$. To remove this ambiguity we use a modified definition where any two mobile monomers $i$ and $j$ are considered to belong to the same string if $j$ replaces $i$ and

$$
i=\arg \min _{\left\{i|| \mathbf{r}_{j}(t)-\mathbf{r}_{i}(0) \mid<\delta\right\}}\left[\left|\mathbf{r}_{j}(t)-\mathbf{r}_{i}(0)\right|\right] .
$$

That is, at any time $t, i$, and $j$ are considered to be in the same string only if the position of $j$ at time $t, \mathbf{r}_{j}(t)$, is within a radius $\delta$ of the old position of $i$, and also $j$ has the shortest distance from $\mathbf{r}_{i}(0)$ when compared to that of all other potentially replacing mobile monomers. Similarly, when $i$ replaces $j$, the monomers $i$ and $j$ are in the same string if

$$
j=\arg \min _{\left\{j|| \mathbf{r}_{i}(t)-\mathbf{r}_{j}(0) \mid<\delta\right\}}\left[\left|\mathbf{r}_{i}(t)-\mathbf{r}_{j}(0)\right|\right] .
$$

Compared to Eq. (8), Eqs. (9) and (10) are more stringent criteria for identifying stringlike motion ${ }^{57}$ However, the new definition and Eq. (8) give the same result for $\delta \leqslant 0.45$. For $\delta=0.6$ we found Y-like ambiguities in $1 \%$, and for $\delta=0.55$ only in $0.2 \%$, of the replacements. Although these ambiguities are relatively uncommon, we wish to avoid them as much as possible while still using a definition close to that used in Ref. 23. Thus, $\delta=0.55$ is chosen for the subsequent analysis.

Once strings are identified at any time $t$, their transient nature and $T$-dependence may be studied by calculating the mean string length. There are two different definitions that may have relevance here. One definition is provided by the weight averaged string length $\langle s(t)\rangle_{w}$, defined by ${ }^{58}$

$$
\langle s\rangle_{w}=\frac{\sum_{s=1}^{\infty} s^{2} P(s)}{\sum_{s=1}^{\infty} s P(s)},
$$

where $s \equiv s(t)$ and $P(s)$ are the string length and the probability of finding a string of length $s$, respectively. This definition is relevant in the context of percolation theory, which was used to analyze clusters of mobile monomers in Ref. 25. For completeness, we present the results for $\langle s(t)\rangle_{w}$ in Appendix A.

A second definition is the number averaged string length $\langle s(t)\rangle$, defined by ${ }^{58}$

$$
\langle s\rangle=\frac{\sum_{s=1}^{\infty} s P(s)}{\sum_{s=1}^{\infty} P(s)},
$$

where $\sum_{s=1}^{\infty} P(s)=1$. This definition arises in the context of equilibrium polymers, which we discuss later in this section as it appears to be relevant to the present study. Thus it is this definition on which we focus in this section. Additionally, in order to investigate whether or not chain connectivity favors the formation of strings, and to understand the interplay of connectivity and mobility, we calculate the number averaged string length $\left\langle s_{\mathrm{seg}}(t)\right\rangle$ of contiguous segments of mobile monomers in a chain. The comparison of the two quantities should reveal the contribution of chain connectivity to the formation of strings.

Figure 7 shows the time evolution of $\langle s(t)\rangle$ and $\left\langle s_{\text {seg }}(t)\right\rangle$. Qualitatively, both quantities behave in the same way. At short times, $\langle s(t)\rangle$ and $\left\langle s_{\text {seg }}(t)\right\rangle$ are equal to one. A

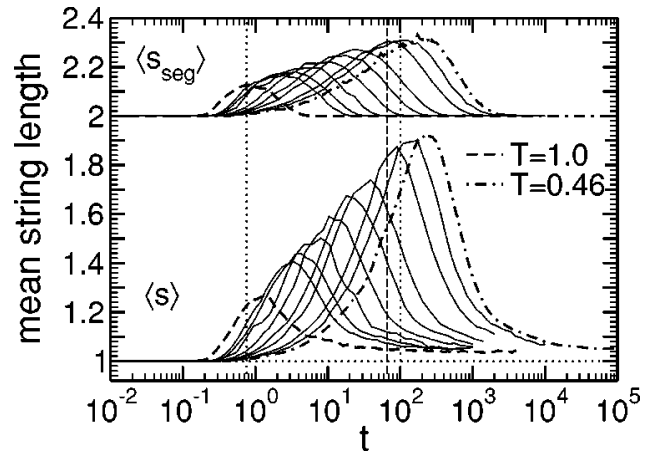

FIG. 7. Average string length $\langle s(t)\rangle$ of all mobile monomers and average string length in contiguous segments of mobile monomers $\left\langle s_{\text {seg }}(t)\right\rangle$ vs $t$ with replacement parameter $\delta=0.55$. The temperatures shown are (from left to right): $T=1,0.7,0.65,0.6,0.55,0.52,0.5,0.48,0.47,0.46$. The dotted vertical lines in the lower panel indicate the time when $\alpha_{2}$ is maximum for $T=1\left(t_{\alpha_{2}}^{*}=0.766\right)$ and $T=0.46\left(t_{\alpha_{2}}^{*}=100.894\right)$, whereas the dashed vertical line indicates the time $t_{\text {clu }}^{\max }$ when the cluster size is maximum for $T$ $=0.46\left(t_{\mathrm{clu}}^{\mathrm{max}}=65.85\right)$. A string length of one corresponds to an isolated mobile monomer, i.e., no "bond" could be formed between two mobile monomers via the replacement criterion of Eqs. (9) and (10). Figure 10 shows that despite the small average string length, large strings containing up to 12 monomers occur with significant probability.

string length of one means that the mobile monomers are separated from each other, and do not replace other mobile monomers when they move. ${ }^{59}$ For a replacement to occur the shortest distance a monomer must travel is the nearestneighbor distance $(\approx 1)$ minus $\delta$, which is roughly 0.45 . Figure 3 shows that such a displacement $\left(g_{0, m} \approx 0.2\right)$ occurs at $t \approx 0.2$ at $T=1$ and $t \approx 2$ at $T=0.46$. These estimates are close to the times at which $\langle s(t)\rangle$ and $\left\langle s_{\text {seg }}(t)\right\rangle$ begin increasing.

A string length larger than one implies that mobile monomers tend to replace each other. This trend is present at all temperatures, but becomes more pronounced on cooling. When compared to the MSD of mobile monomers $g_{0, m}$ the peak time $t_{\mathrm{str}}^{\max }$ for $\langle s(t)\rangle$ corresponds to a time where $g_{0, m}$ $\approx 1$ [see Fig. 3(a) or 3(b)]. Thus, $\langle s(t)\rangle$ is maximum when a mobile monomer moves on average a distance equal to its size. With respect to the MSD of the bulk, this time corresponds to the time when there is a crossover from a caging regime to a subdiffusive regime, similar to what is observed for other dynamical quantities as discussed in earlier sections.

For times larger than $t_{\mathrm{str}}^{\max },\left\langle s_{\mathrm{seg}}(t)\right\rangle$ relaxes back to its initial value of one, whereas $\langle s(t)\rangle$ asymptotically tends to a slightly larger value $(\sim 1.04)$. This disparity is related to the definitions of $\langle s(t)\rangle$ and $\left\langle s_{\text {seg }}(t)\right\rangle$ which give rise to a different large- $t$ limit in the diffusive regime (see Appendix B). Notice that the $t_{\mathrm{str}}^{\max }$ occurs at a slightly later time than $t_{\mathrm{clu}}^{\max }$. In principle, one would expect the clusters of mobile monomers and the strings they are comprised of to be maximum at roughly the same time. This apparent discrepency is currently being investigated. ${ }^{60}$ In the present system, finite size effects may occur at low $T$, where the clusters become larger than the simulation cell. This could give an estimate of $t_{\text {clu }}^{\max }$ different from the asymptotic value. It is also possible that in the present systems, $t_{\mathrm{str}}^{\max }$ is larger than $t_{\mathrm{clu}}^{\max }$ because there may be a time delay for mobile particles to rearrange them- 


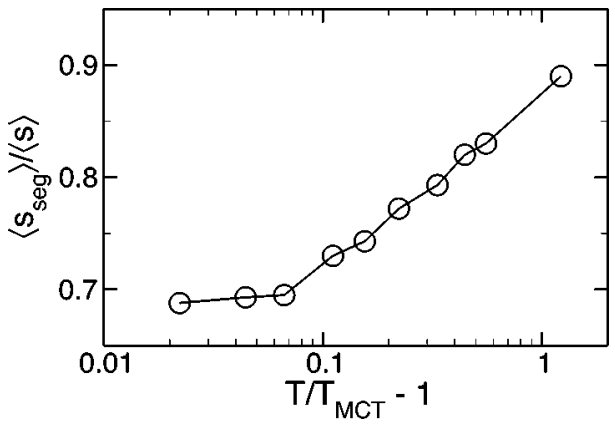

FIG. 8. Temperature dependence of the ratio of $\left\langle s_{\mathrm{seg}}\left(t_{\mathrm{str}}^{\max }\right)\right\rangle$ and $\left\langle s\left(t_{\mathrm{str}}^{\max }\right)\right\rangle$. $t_{\text {str }}^{\max }$ is the peak time of $\left\langle s_{\text {seg }}\right\rangle$ and $\langle s\rangle$ at different temperatures. $T_{\mathrm{MCT}}$ $=0.45$.

selves in a special one-dimensional path. In any case, despite this small difference in the peak times, both $t_{\mathrm{str}}^{\max }$ and $t_{\mathrm{clu}}^{\max }$ occur in the late- $\beta /$ early- $\alpha$ relaxation regime when particles begin to break out of their cage. (The different relaxation times discussed in this work are compiled in Appendix $\mathrm{C}$ and their dependence on temperature is compared.)

To understand the role of chain connectivity on strings, we compare $\left\langle s_{\text {seg }}\left(t_{\text {str }}^{\max }\right)\right\rangle$ and $\left\langle s\left(t_{\text {str }}^{\max }\right)\right\rangle$ in Fig. 8 by taking the ratio of the two quantities. A ratio near one (unless both the numerator and denominator are equal to one) implies that the strings are the result of consecutively bonded pairs, i.e., that monomers moving in strings actually move along the backbone of the chain to which they belong, indicating an important contribution from connectivity to the formation of strings. On the other hand, a ratio close to zero implies that stringlike motion occurs among nonbonded monomers and that chain connectivity is insignificant. As indicated in the figure, $\left\langle s_{\mathrm{seg}}\right\rangle /\langle s\rangle$ decreases as $T$ approaches $T_{\mathrm{MCT}}$, suggesting that chain connectivity becomes less important for stringlike motion at low $T$.

The previous analysis was performed with $\delta=0.55$. When introducing the criterion for defining strings we argued that the precise choice of $\delta$ is not crucial, as long as its value is sufficiently small. To illustrate this point, Fig. 9 shows the temperature dependence of the maximum average string length, $\left\langle s\left(t_{\mathrm{str}}^{\mathrm{max}}\right)\right\rangle$, for various $\delta$. We find that the strings become longer if $\delta$ increases. This is expected, since more particles satisfy the $|\cdot|<\delta$ condition. However, the qualitative features are independent of $\delta$. To support this point further we invoke an analogy, first proposed in Ref. 23, between the strings and equilibrium polymers ${ }^{61-63}$ (see also Ref. 64 and references therein).
Equilibrium polymers are systems in which the bonds between monomers are not permanent. They can constantly break and recombine at random points along the backbone of a chain. In chemical equilibrium a melt of these selfassembling polymers is characterized by an exponential distribution of chain lengths, $P(s) \sim \exp (-s /\langle s\rangle)$ (if $s$ is large), and by a mean chain length that increases exponentially with the energy $E$ gained by bond formation, $\langle s\rangle \propto \exp (E / T)$.

In our context, the mobile monomers also self-assemble into chains, driven by the sluggish dynamics of the cold melt. The dynamically created bonds can break and recombine at any instant. They are more likely to form, and thus "stronger," the larger the choice of $\delta$. This suggests a correspondence between $\delta$ and $E$, the simplest assumption being $E \propto \delta$. Figure 9 shows that this assumption is not unrealistic. Despite the disparity between the theoretical premise of long chains and the relative shortness of our strings, a reasonable superposition of string lengths, found for various $\delta$ and $T$, is obtained. This implies that any of the values for $\delta$ presented could have been chosen for the present analysis.

From the analogy with equilibrium polymers one expects that the strings have an exponential distribution. Figure 10 shows the distribution of the string length $s$ found at $t_{\mathrm{str}}^{\max }$. At the highest temperature, $T=1, P(s)$ is an exponential and decreases rapidly with increasing $s$. The most frequent string lengths are $s=1,2$. Their probability remains essentially unchanged on cooling, whereas longer strings occur much more frequently for $T<1$. The tail of the distribution appears to remain exponential, further supporting the possible interpretation of strings in the same context as equilibrium polymers. Similar observations of exponential distributions were also made in simulations of a binary LJ mixture. ${ }^{23}$

The weight averaged string length $\langle s(t)\rangle_{w}$ is presented in Appendix A. We find that $\langle s(t)\rangle_{w}$ and the number average $\langle s(t)\rangle$ behave qualitatively in the same way, but are quantitatively different. This difference characterizes the variance of the string length distribution because

$$
\frac{\left\langle(s-\langle s\rangle)^{2}\right\rangle}{\langle s\rangle^{2}}=\frac{\langle s\rangle_{w}}{\langle s\rangle}-1,
$$

where $\langle\cdot\rangle$ denotes the number average [see Eq. (12)]. The ratio $\langle s\rangle_{w} /\langle s\rangle$, shown in Fig. 11, is referred to as the "polydispersity index" in the context of polymerization. We find that the strings are most polydisperse for times of the late- $\beta$ / early- $\alpha$ process and that this maximum of polydispersity increases with decreasing $T$.

In carrying out this analysis, the question arises as to whether a string of length one should be included or not in
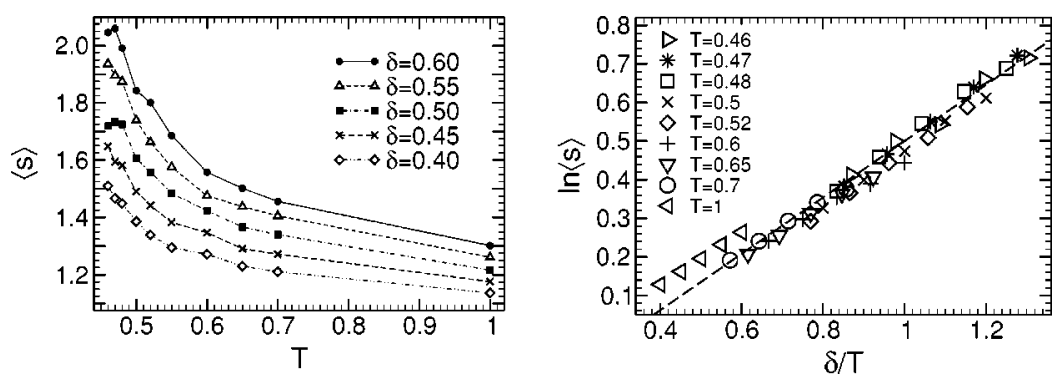

FIG. 9. Left panel: average string length $\langle s\rangle$ vs $T$ for various $\delta$ [see Eqs. (9) and (10)]. The string length is calculated at $t_{\mathrm{str}}^{\max }$, where it is maximum. Right panel: rescaling of $\langle s\rangle$ as suggested by the analogy with equilibrium polymers (see text for details). A satisfactory collapse of the data for all $T$ and $\delta$ is obtained except for $T=1$, the temperature below which supercooled liquid dynamics occurs in this model. At this temperature, strings larger than 1 occur very seldomly (see Fig. 10). The dashed straight line is a fit through the data for $T$ $\leqslant 0.7$, yielding $\ln \langle s\rangle=-0.23+0.73 \delta / T$. 


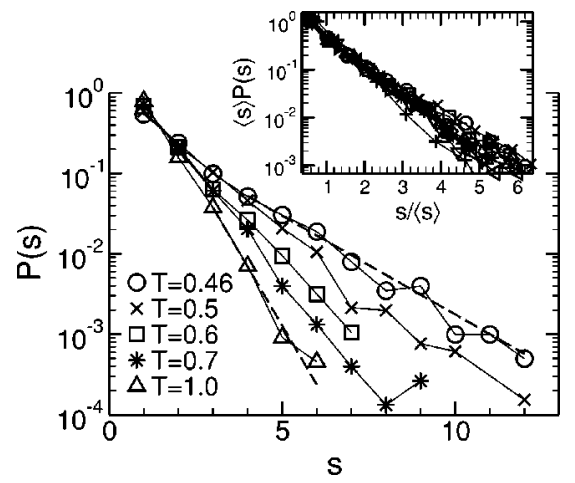

FIG. 10. Semi-log plot of the probability distribution $P(s)$ of the string length $s$ for various $T$. $P(s)$ is calculated at $t=t_{\mathrm{str}}^{\max }$ where $\langle s\rangle$ is maximum. All data sets exhibit (roughly) exponential behavior. Inset: $P(s)$ rescaled by the mean value $\langle s\rangle$ vs $s /\langle s\rangle$. In addition to the temperatures $T=0.46,0.47$, $0.48,0.50,0.52,0.55$ for $\delta=0.55$ the graph also includes $T=0.46$ and 0.55 for both $\delta=0.4$ and $\delta=0.6$. The scaling deteriorates if data at higher $T$ is included.

the calculation of mean string lengths. One may argue that $s=1$ should be excluded from the calculation since by definition stringlike motion requires one mobile monomer to replace another, and hence is not really defined for $s<2$. In other words, a "string" of length one indicates a mobile monomer not moving in a string based on the criterion used. To address this issue, we calculate the time evolution of $\langle s(t)\rangle$ by excluding strings of size one (see Appendix A), for comparison. We find no qualitative difference between the two cases, but in the absence of $s=1$ the mean string length is accordingly larger. Therefore, to maintain the analogy of strings to equilibrium polymerization, in which polymers of length one are included, we restrict our discussion to the mean values that include $s=1$ and are calculated using the number average.

\section{E. Directional correlations between neighboring mobile monomers}

The above analysis shows that mobile monomers follow each other, replacing one another in one-dimensional paths

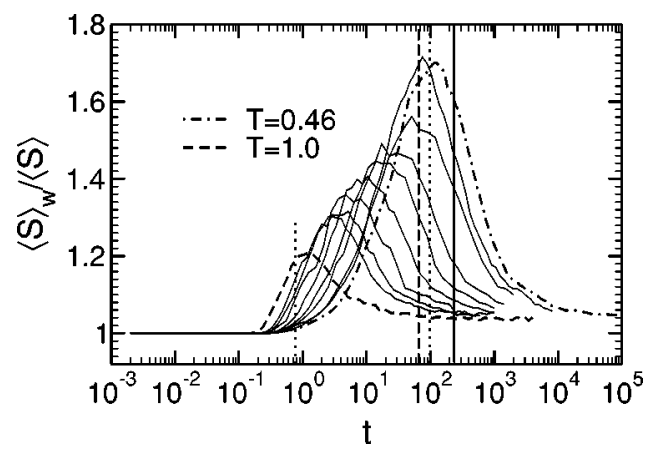

FIG. 11. The "polydispersity index" given by the ratio of the weight averaged string length $\langle s(t)\rangle_{w}$ and the number averaged string length $\langle s(t)\rangle$ plotted as a function of time for all temperatures. The temperatures shown are (from left to right): $T=1,0.7,0.65,0.6,0.55,0.52,0.5,0.48,0.47,0.46$. The vertical dotted lines indicate $t_{\alpha_{2}}^{*}=0.766$ and $t_{\alpha_{2}}^{*}=100.894$ for $T=1$ and $T=0.46$, respectively. For $T=0.46$ the vertical dashed line shows $t_{\text {clu }}^{\max }(=65.85)$, the vertical solid $t_{\text {str }}^{\max }(=236.26)$.
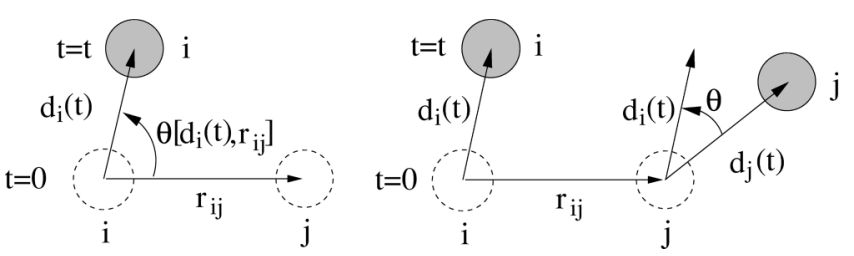

FIG. 12. Left panel: Definition of $\theta\left[\mathbf{d}_{i}(t), \mathbf{r}_{i j}\right]$, Eq. (16). Right panel: Definition of $\theta\left[\mathbf{d}_{i}(t), \mathbf{d}_{j}(t)\right]$, Eq. (17). In both panels, the dashed circles depict the position of the mobile monomers $i$ and $j$ at $t=0$, whereas the shaded circles represent their positions at time $t$. The vectors $\mathbf{r}_{i j}$ and $\mathbf{d}_{i}(t)$ are defined by Eqs. (14) and (15), respectively.

and forming strings of different sizes whose mean value is a maximum at a time $t_{\mathrm{str}}^{\max }$. Since typical displacements of mobile monomers in this time regime are still fairly local (see Fig. 3), a replacement is most likely to occur between nearest neighbors. In the remaining part of this section, we thus concentrate on neighboring mobile monomers and explore their correlated motion in further detail. In particular, we address the directional aspect of correlations between mobile monomers that are bonded or nonbonded.

Let monomers $i$ and $j$ be nearest neighbors at $t=0$ and mobile at some later time $t$. We define $i$ to be a nearest neighbor of $j$ if their initial distance is within the first neighbor shell of the pair-distribution function, i.e., $\mid \mathbf{r}_{j}(0)$ $-\mathbf{r}_{i}(0) \mid<1.5 .^{35}$ The same definition was also used in Ref. 25 . To simplify the notation we write for the distance between two neighbors at time $t=0$

$$
\mathbf{r}_{i j}=\mathbf{r}_{j}(0)-\mathbf{r}_{i}(0),
$$

and for the displacement of monomer $i$ in time $t$

$$
\mathbf{d}_{i}(t)=\mathbf{r}_{i}(t)-\mathbf{r}_{i}(0) .
$$

We can define the following angles:

$$
\theta\left[\mathbf{d}_{i}(t), \mathbf{r}_{i j}\right]=\arccos \left[\frac{\mathbf{d}_{i}(t) \cdot \mathbf{r}_{i j}}{\left|\mathbf{d}_{i}(t)\right|\left|\mathbf{r}_{i j}\right|}\right], \quad \theta\left[\mathbf{d}_{i}(t), \mathbf{r}_{i j}\right] \in[0, \pi]
$$

and

$$
\begin{aligned}
\theta\left[\mathbf{d}_{i}(t), \mathbf{d}_{j}(t)\right]= & \arccos \left[\frac{\mathbf{d}_{i}(t) \cdot \mathbf{d}_{j}(t)}{\left|\mathbf{d}_{i}(t) \| \mathbf{d}_{j}(t)\right|}\right], \\
& \theta\left[\mathbf{d}_{i}(t), \mathbf{d}_{j}(t)\right] \in[0, \pi] .
\end{aligned}
$$

These definitions are illustrated in Fig. 12. The first angle [Eq. (16)] was proposed in Ref. 23. It indicates to what extent a mobile monomer is displaced, at time $t$, in a direction parallel to a vector connecting its initial position to that of one of its neighbors which is also mobile at time $t$. The second angle [Eq. (17)] measures the correlations between the displacements $\mathbf{d}_{i}(t)$ and $\mathbf{d}_{j}(t)$ of the monomers at time $t$. Thus, it shows the extent of directional correlation between any two neighboring mobile monomers. For both bonded and nonbonded nearest-neighbor pairs we computed the probability distributions $P_{d, r}\left(\theta\left[\mathbf{d}_{i}(t), \mathbf{r}_{i j}\right]\right)$ and $P_{d, d}\left(\theta\left[\mathbf{d}_{i}(t), \mathbf{d}_{j}(t)\right]\right)$ normalized by the probability for isotropic, uncorrelated displacements ${ }^{65}$

$$
P_{\text {iso }}(\theta)=\frac{1}{2} \sin (\theta) \text {. }
$$




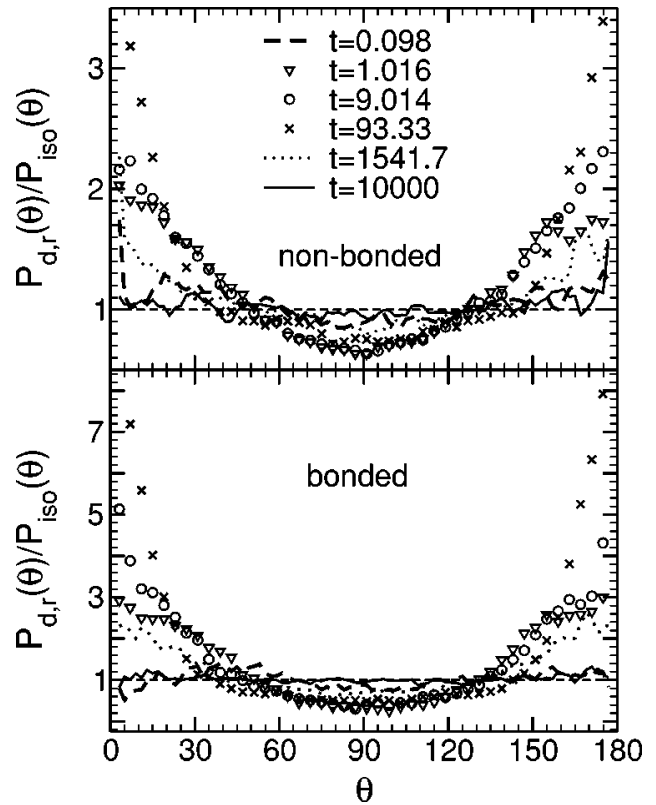

FIG. 13. Probability distribution $P_{d, r}\left(\theta\left[\mathbf{d}_{i}(t), \mathbf{r}_{i j}\right]\right)$ of the angle between the displacement vector of a mobile monomer $\mathbf{d}_{i}(t)$ and of the vector $\mathbf{r}_{i j}$ between the initial positions of the monomer and of its mobile neighbor $j . P_{d, r}$ is divided by the probability $P_{\text {iso }}$ for isotropic motion. The upper figure presents the results for bonded nearest neighbors, the lower for nonbonded nearest neighbors. In both cases, $T=0.47$. The times shown are separated from each other roughly by a factor of $10: t=0.098(\approx$ first maximum of $\left.f_{e, m}\right), \quad t=1.016 \quad$ (beginning of the $\beta$-regime), $t=9.014$ (center of the $\beta$-regime, where $\left.g_{0} \simeq 6 r_{0, c}^{2}\right), t=93.33\left(=t_{\mathrm{seg}}^{\max }\right), t=1541.7\left(=t_{\mathrm{seg}}^{\min }\right)$, $t=10^{4}\left(\hat{=} g_{0}>R_{g}^{2}\right)$.

Figure 13 shows the ratio $P_{d, r}(\theta) / P_{\text {iso }}(\theta)$ at $T=0.47$ for several selected times (the times are approximately uniformly distributed on a logarithmic scale.) At the earliest and the latest time, we find $P_{d, r}(\theta) \approx P_{\text {iso }}(\theta)$, consistent with the expectation that the motion should be directionally uncorrelated in these limits. This behavior persists up to approximately $t \approx 0.1$ for the short-time regime. For intermediate times, displacements perpendicular to the axis $\mathbf{r}_{i j}$ are suppressed relative to $P_{\text {iso }}$, whereas motion parallel to it is enhanced as indicated by large values near $\theta=0$ and $\theta=180$ $\left[P_{d, r}(\theta)\right.$ is symmetric $\left.{ }^{66}\right]$. This directional preference is particularly prominent in the late- $\beta /$ early- $\alpha$ regime: as the monomers escape from their cages, they tend to replace a neighbor. Similar findings were reported in Ref. 23 for a $\mathrm{LJ}$ mixture. Comparison between Figs. 13(a) and 13(b) shows that this directional correlation is more pronounced for bonded mobile monomers.

To ascertain the influence of temperature on the time evolution of this directionally correlated motion, we find that a direct comparison of the probability distributions obscures the details, since the graphs become fairly crowded and hide the main trends. Thus another averaged quantity, which is sensitive to preferential motion in the direction parallel to $\mathbf{r}_{i j}$, is proposed. One choice is

$$
\begin{aligned}
C_{d, r}(t)= & \frac{1}{\pi} \int_{0}^{\pi}\left|\cos \left(\theta\left[\mathbf{d}_{i}(t), \mathbf{r}_{i j}\right]\right)\right| \\
& \times \frac{P_{d, r}\left(\theta\left[\mathbf{d}_{i}(t), \mathbf{r}_{i j}\right]\right)}{P_{\text {iso }}\left(\theta\left[\mathbf{d}_{i}(t), \mathbf{r}_{i j}\right]\right)} \mathrm{d} \theta\left[\mathbf{d}_{i}(t), \mathbf{r}_{i j}\right]-\frac{2}{\pi} .
\end{aligned}
$$

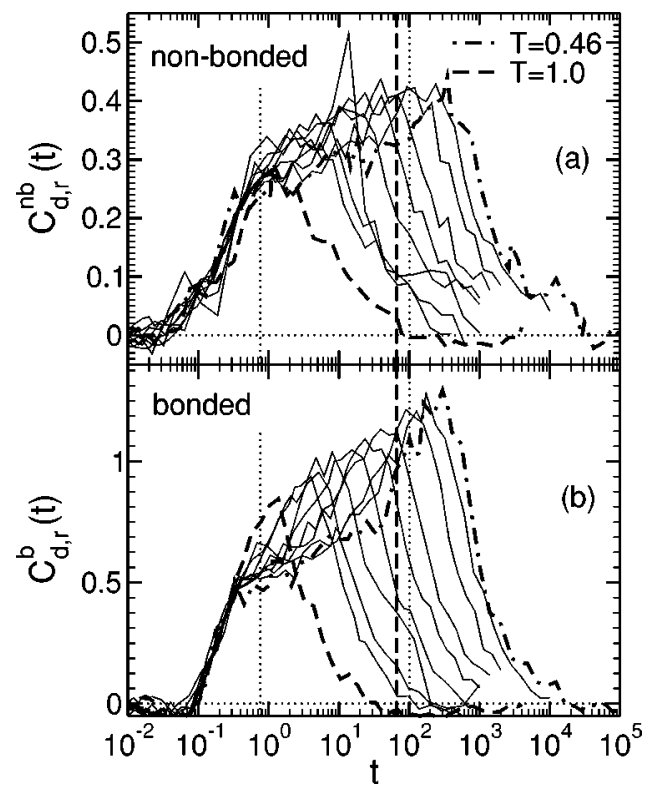

FIG. 14. $C_{d, r}(t)$ vs time for all $T$. $C_{d, r}$ measures the tendency of a mobile particle to replace a mobile nearest neighbor at time $t$ [Eq. (19)]. (a) and (b) present the results for nonbonded and bonded nearest neighbors. In both panels, the vertical dotted lines indicate the times $t_{\alpha_{2}}^{*}$ for $T=1\left(t_{\alpha_{2}}^{*}\right.$ $=0.766)$ and for $T=0.46\left(t_{\alpha_{2}}^{*}=100.894\right)$, whereas the dashed vertical line indicates the time $t_{\mathrm{clu}}^{\max }$ when mean cluster size, calculated in Ref. 25 , is maximum for $T=0.46\left(t_{\mathrm{clu}}^{\max }=65.85\right)$. The following temperatures are shown (curves from left to right): $T=1,0.7,0.65,0.6,0.55,0.52,0.5,0.48,0.47$, 0.46 .

We use the absolute value of the cosine because the probability distribution is symmetric about $90^{\circ}$. In order to set $C_{d, r}(t)=0$ for isotropically distributed displacement directions, we subtract $(1 / \pi) \int_{0}^{\pi}|\cos \theta| \mathrm{d} \theta=2 / \pi$. The resulting quantity measures deviations, in terms of directional correlations, from isotropic behavior and enables us to perform a time-resolved comparison for different $T$. Note that Eq. (19) is not an expectation value because the ratio $P_{d, r} / P_{\text {iso }}$ is not a probability distribution. If $\mathbf{d}_{i}$ and $\mathbf{r}_{i j}$ are perfectly parallel to each other, $C_{d, r}$ diverges.

Figure 14 depicts the time and temperature dependence of $C_{d, r}$ for both bonded and nonbonded nearest neighbors. Several observations can be inferred from this figure: First, for all $T$ the displacement of the mobile monomers is isotropic in the ballistic regime and for times in the $\alpha$-regime provided that $d_{i} \gg 1.5$ (for $T=1$ and 0.46 this implies $t>10$ and $t>10^{4}$, respectively; see Fig. 3). Second, at intermediate times the motion of mobile monomers shows an enhanced tendency to replace a nearest neighbor. This tendency is present at all $T$, but increases on cooling toward $T_{\mathrm{MCT}}$. It is maximum in the late- $\beta /$ early- $\alpha$ regime. Third, the propensity of displacements along the nearest-neighbor axis is more pronounced if the neighbor is directly bonded to the mobile monomer. These findings concur with those of Fig. 13, but in addition demonstrate the $T$-dependence.

For $\theta\left[\mathbf{d}_{i}(t), \mathbf{d}_{j}(t)\right]$ we define a similar quantity to measure the tendency of mobile neighbors to follow each other at time $t$ 


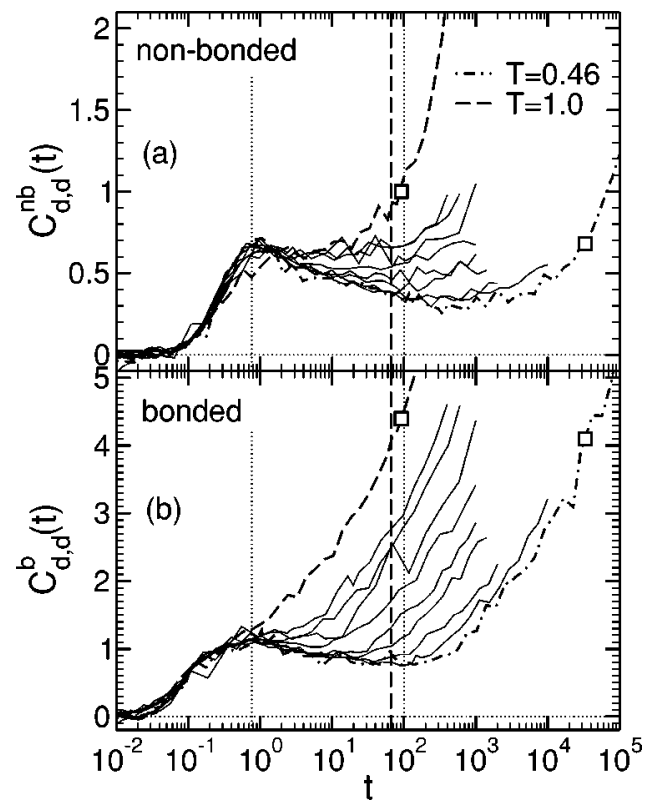

FIG. 15. $C_{d, d}(t)$ vs time for all $T$. $C_{d, d}$ measures the tendency of two neighboring mobile particles to follow each other at time $t$ [Eq. (20)]. (a) and (b) present the results for nonbonded and bonded nearest neighbors. In both panels, the vertical dotted lines indicate the times $t_{\alpha_{2}}^{*}$ for $T=1\left(t_{\alpha_{2}}^{*}\right.$ $=0.766)$ and for $T=0.46\left(t_{\alpha_{2}}^{*}=100.894\right)$, whereas the open squares mark the times when $g_{e} / g_{0}$ reaches its second maximum ( $t \simeq 92$ for $T=1, t$ $\simeq 33361$ for $T=0.46$; see Fig. 6 ). The dashed vertical line in both panels indicates the time $t_{\text {clu }}^{\max }$ when mean cluster size, calculated in Ref. 25, is maximum for $T=0.46\left(t_{\mathrm{clu}}^{\max }=65.85\right)$. The following temperatures are shown (curves from left to right): $T=1,0.7,0.65,0.6,0.55,0.52,0.5,0.48,0.47$, 0.46 .

$$
\begin{aligned}
C_{d, d}(t)= & \frac{1}{\pi} \int_{0}^{\pi} \cos \left(\theta\left[\mathbf{d}_{i}(t), \mathbf{d}_{j}(t)\right]\right) \\
& \times \frac{P_{d, d}\left(\theta\left[\mathbf{d}_{i}(t), \mathbf{d}_{j}(t)\right]\right)}{P_{\text {iso }}\left(\theta\left[\mathbf{d}_{i}(t), \mathbf{d}_{j}(t)\right]\right)} \mathrm{d} \theta\left[\mathbf{d}_{i}(t), \mathbf{d}_{j}(t)\right],
\end{aligned}
$$

which gives zero for uncorrelated motion and diverges to $+\infty$ if the mobile neighbors follow each other in perfect alignment (or to $-\infty$ for antiparallel motion).

Figure 15 depicts $C_{d, d}(t)$ for all temperatures. Beyond the ballistic regime $C_{d, d}(t)$ is positive for both bonded and nonbonded mobile monomers. The positive value implies that small angles between the displacements $\mathbf{d}_{i}(t)$ and $\mathbf{d}_{j}(t)$ are more likely than expected for isotropic motion. ${ }^{67}$ Thus, mobile monomers have an enhanced tendency to follow each other for all times outside the ballistic regime. This tendency is particularly pronounced at $t \approx 1$, which corresponds to the early $\beta$-relaxation at low temperatures, and for $t \gg t_{\alpha_{2}}^{*}$, where the monomer displacements are determined first by chain connectivity and later by the diffusion of the center-of-mass. In the diffusive regime one expects $C_{d, d}(t)$ to be large. After large times many monomers have moved in similar directions so that the center-of-mass advances substantially. Much before the diffusive regime, however, $C_{d, d}(t)$ first "grows" and then "shrinks" as the observation time increases. A clue to interpret this behavior is obtained by calculating the ratio of the bonded $(b)$ and the nonbonded $(n b)$ neighbors. We find that $C_{d, d}^{b}(t) / C_{d, d}^{n b}(t) \sim g_{e}(t) / g_{0}(t)$ (see Fig. 6 for com-

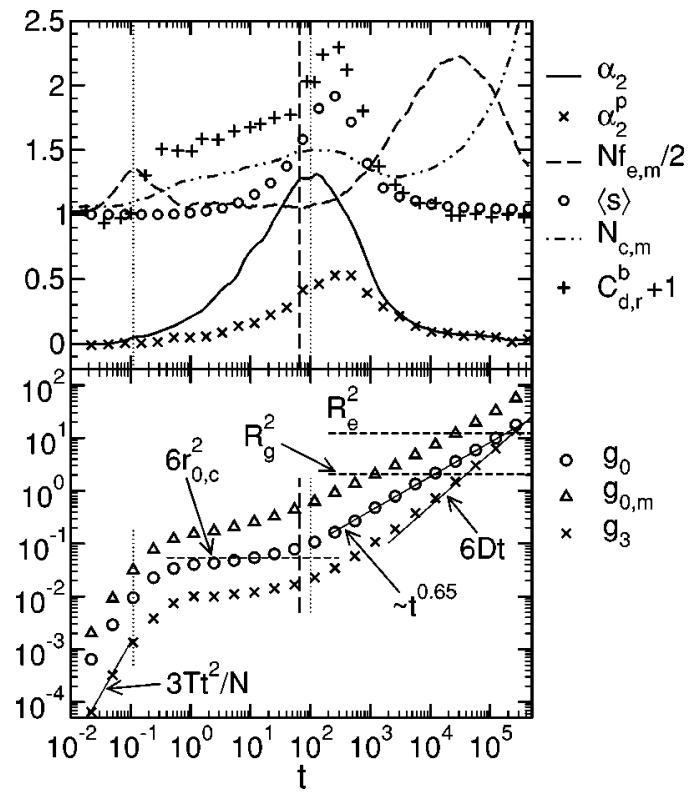

FIG. 16. Recapitulating the comparison of the time dependence of various quantities discussed in this paper. In all cases, the temperature is $T=0.46$ $\left(T_{\mathrm{MCT}} \simeq 0.45\right)$. The upper figure shows the non-Gaussian parameters $\alpha_{2}$ and $\alpha_{2}^{p}$. These quantities are averages over all monomers and all chains in the melt. The other quantities shown are calculated from the $6.5 \%$ of highly mobile monomers. They are: the fraction of mobile end-monomers $N f_{e, m} / 2$, the average string length $\langle s\rangle$, the average length of mobile contiguous segments of a chain $N_{c, m}$, and the cosine of the angle between the displacement of a mobile monomer and the vector to one of its nearest mobile neighbors in the chain $C_{d, r}^{b}$. The vertical dotted lines indicate the time of the first peak of $N f_{e, m} / 2(t=0.1104)$, the time $t_{\alpha_{2}}^{*}(=100.894)$ where $\alpha_{2}$ is maximum, whereas the vertical dashed line indicates the time $t_{\text {clu }}^{\max }$ when the clusters formed by the subset of mobile monomers is largest for $T=0.46$ ( $t_{\text {clu }}^{\max }$ $=65.85$ ) (Ref. 25). These times are also included in the lower figure (vertical dotted resp. dashed lines). This figure shows the MSD of all monomers $g_{0}$, of the mobile monomers $g_{0, m}$, and of the center-of-mass $g_{3}$. The behavior in the ballistic $\left(\sim t^{2}\right)$, the subdiffusive $\left(\sim t^{0.65}\right)$ and the diffusive regimes $(\sim t)$ are indicated by solid lines. The horizontal dashed lines depict $6 r_{0, c}^{2}$ (Lindemann localization length $r_{0, c} \simeq 0.095$ for $g_{0}$ ), the radius of gyration $R_{g}^{2}(=2.09)$, and the end-to-end distance $R_{e}^{2}(=12.3)$.

parison). The difference between $C_{d, d}^{b}(t)$ and $C_{d, d}^{n b}(t)$ is large for $t \approx 0.1$ and for $t_{\alpha_{2}}^{*} \lesssim t \lesssim \tau_{\mathrm{R}}$, where the mean-square displacement of the end monomers is enhanced compared to the average displacement. It is plausible that a highly mobile end monomer will trigger large displacements of the neighbor connected to it and bias this displacement in the direction of its own motion. This effect is strongly suppressed in the $\beta$-regime, where $g_{e}(t)$ and $g_{0}(t)$ are alike. For $1 \leqq t \lesssim t_{\alpha_{2}}^{*}$, $C_{d, d}^{b}(t) / C_{d, d}^{n b}(t) \approx$ constant, comparable to $g_{e} / g_{0}$.

\section{SUMMARY AND CONCLUSIONS}

We presented a detailed analysis of the local dynamics of a simulated equilibrium polymer melt approaching its glass transition. Our analysis utilizes the $6.5 \%$ of monomers with the largest displacement at some time $t$. These monomers are called "mobile." For this subensemble, we addressed the following questions: (1) Do the mobile monomers follow each other in a stringlike fashion, as observed for a binary LJ- 

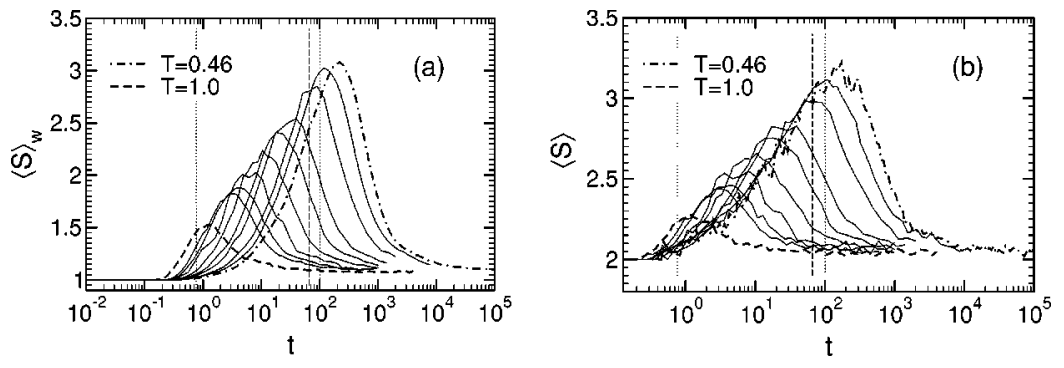

FIG. 17. (a) Weight averaged string length $\langle s(t)\rangle_{w}$ of all mobile monomers vs $t$. (b) Number averaged string length $\langle s(t)\rangle$ of all mobile monomers vs $t$ calculated by excluding strings of size 1 . In both figures, the temperatures shown are (from left to right): $T=1,0.7,0.65,0.6$, $0.55,0.52,0.5,0.48,0.47,0.46$. The dotted vertical lines in both panels indicate the time when $\alpha_{2}$ is maximum for $T=1 \quad\left(t_{\alpha_{2}}^{*}=0.766\right)$ and $T=0.46 \quad\left(t_{\alpha_{2}}^{*}\right.$ $=100.894)$. The dashed vertical line indicates the time $t_{\text {clu }}^{\max }$ when the weight averaged cluster size, calculated in Ref. 25, is maximum for $T=0.46\left(t_{\text {clu }}^{\max }=65.85\right)$. mixture? (2) If yes, what are the properties of these dynamic strings? (3) What is the influence of chain connectivity on this correlated motion?

The answers to these questions may be summarized as follows (see Fig. 16):

There is an intermediate time interval in which stringlike motion is most pronounced. In the cold melt this interval corresponds to the late- $\beta$ /early- $\alpha$ process; the average string length $\langle s(t)\rangle$ is maximum at a time $t_{\mathrm{str}}^{\max }$ that is slightly later than the time when clusters formed by the mobile particles are largest. ${ }^{25}$ Close to $T_{\text {MCT }}$, the temperature dependence of $t_{\mathrm{str}}^{\mathrm{max}}$ is approximately the same as that of the inverse diffusion coefficient $1 / D$ (the product $D t_{\text {str }}^{\max } \approx$ const, see Appendix C), which was shown in Ref. 31 to scale differently from the $\alpha$-relaxation time when fitted to the power law of the MCT. The similarity in the scaling between $t_{\mathrm{str}}^{\max }$ and $1 / D$ is not unexpected, because $t_{\mathrm{str}}^{\max }$ is extracted from the dynamics of monomers with the largest displacements, while these monomers are most likely the ones that determine the diffusion behavior, and hence $D$;

(iii) the maximum string length $\left\langle s\left(t_{\mathrm{str}}^{\max }\right)\right\rangle$ increases with decreasing $T$, approximately in an exponential fashion (Fig. 9). The distribution of string lengths $s$ at any $T$ is roughly exponential (Fig. 10). These findings are identical to those observed in a LJ mixture, ${ }^{23}$ and suggest an analogy between strings and equilibrium polymers, as proposed in Ref. 23;

(iv) the average string length remains fairly small on cooling. Even at $T=0.46,\left\langle s\left(t_{\text {str }}^{\max }\right)\right\rangle \approx 2$ (calculated by including $s=1$ ), although strings as long as 12 monomers are observed;

(v) an important contribution to correlated motion comes from chain connectivity. A monomer tends to replace one of its bonded neighbors (see Fig. 7 and $C_{d, r}^{b}$ in Fig. 16). However, mobility is not concentrated along the backbone of some chains. If this was the case, the average string length calculated for the monomers of a chain only, $\left\langle s_{\text {seg }}\right\rangle$ (Fig. 7), and the average length of mobile contiguous segments, $N_{c, m}$, should be of the order of the chain length $N$. Therefore, a relaxation mechanism in which mobile monomers are connected to each other and slide along the backbone of the chain is unlikely.

\section{ACKNOWLEDGMENTS}

The authors are grateful to M. Fuchs and J. F. Douglas for many helpful discussions. We gratefully acknowledge generous amounts of computing time by the Center for Theoretical and Computational Material Science at NIST, Gaithersburg, and the computer center at the Johannes GutenbergUniversity Mainz. Financial support by the SFB262, the DAAD ("Doktorandenstipendium im Rahmen des gemeinsamen Hochschulsonderprogramms III von Bund und Ländern," Grant No. D/00/07994), the BMBF (Grant No. D.I.P. 352-101), and the ESF Program on "Experimental and Theoretical Investigation of Complex Polymer Structures" (SUPERNET) is gratefully acknowledged.

\section{APPENDIX A: WEIGHT AND NUMBER AVERAGED STRING LENGTHS}

To compare the weight averaged string length $\langle s(t)\rangle_{w}$ with the number averaged string length $\langle s(t)\rangle$, discussed in Fig. 7, we show here the time evolution of $\langle s\rangle_{w}$, which is calculated using Eq. (11), for different $T$. It is apparent from the figure [Fig. 17(a)] that $\langle s(t)\rangle_{w}$ is qualitatively the same as $\langle s(t)\rangle$.

To address the issue of including or excluding strings of size $s=1$, in Fig. 17(b) we show the number averaged string length $\langle s(t)\rangle$ for several $T$ calculated by excluding $s=1$. This calculation yields a similar result to that obtained by including strings with size $s=1$, but the number average and the weight average (not shown) take larger values when $s$ $=1$ is excluded [compare Figs. 7 and 17(b)].

\section{APPENDIX B: LARGE TIME LIMIT OF THE NUMBER AVERAGED STRING LENGTHS}

For long times the average string length of contiguous segments of mobile monomers, $\left\langle s_{\text {seg }}(t)\right\rangle$, tends to 1 , whereas the average string length of all mobile monomers, $\langle s(t)\rangle$, approaches a slightly larger value $(\sim 1.04)$ (see Figs. 4 and 7 for an illustration of the string lengths). We can explain this difference by the following argument: Let $P_{\delta}\left(\mid \mathbf{r}_{i}(t)\right.$ $-\mathbf{r}_{j}(0) \mid<\delta$ ) denote the probability that a mobile monomer $i$ at time $t$ approaches the initial position of another mobile monomer $j$ to within the range $\delta$. Then, $(0.065 M-1) P_{\delta}$ is the average number of mobile monomers that satisfy the criterion of string formation. Hence, the average string length is given by

$$
\langle s(t)\rangle=1+(0.065 M-1) P_{\delta} .
$$


For $P_{\delta}$ we can write

$$
\begin{aligned}
P_{\delta}= & \int_{\varepsilon<\delta} \mathrm{d} \varepsilon\left\langle\delta\left(\left[\mathbf{r}_{i}(t)-\mathbf{r}_{j}(0)\right]-\varepsilon\right)\right\rangle \\
= & \int_{\varepsilon<\delta} \mathrm{d} \varepsilon\left\{\int \mathrm{d} \mathbf{r}_{i}(t) \mathrm{d} \mathbf{r}_{j}(0) \delta\left(\left[\mathbf{r}_{i}(t)-\mathbf{r}_{j}(0)\right]-\varepsilon\right)\right. \\
& \left.\times G_{s}\left(\mathbf{r}_{i}(t)-\mathbf{r}_{i}(0), t\right)\left[\frac{\rho}{M} g\left(\mathbf{r}_{i}(0)-\mathbf{r}_{j}(0)\right)\right]\right\}(t \text { large }),
\end{aligned}
$$

where $G_{s}(\mathbf{r}, t)$ and $g(\mathbf{r})$ are the self-part of the van Hove correlation function and the pair-distribution function, respectively. Since $\rho g\left(\mathbf{r}_{i j}\right) / M$ is the probability density that the two monomers are initially at a distance $\mathbf{r}_{i j}=\mathbf{r}_{i}(0)$ $-\mathbf{r}_{j}(0)$, and $G_{s}\left(\mathbf{r}_{i}(t)-\mathbf{r}_{i}(0), t\right)$ is the probability density for the displacement $\mathbf{r}_{i}(t)-\mathbf{r}_{i}(0)$, the product $\rho g G_{s} / M$ gives the probability density for the vector $\mathbf{r}_{i}(t)-\mathbf{r}_{j}(0)$, provided the displacement of monomer $j$ is not correlated with $\mathbf{r}_{i}(0)$. This condition can only be valid for large times. If we perform the integral over the $\delta$-function and use the homogeneity of space, we obtain

$$
P_{\delta}=\frac{\rho}{M} \int_{\varepsilon<\delta} \mathrm{d} \varepsilon \int \mathrm{d} \mathbf{r}_{i j} G_{s}\left(\mathbf{r}_{i j}+\varepsilon, t\right) g\left(\mathbf{r}_{i j}\right) \quad(t \text { large }) .
$$

In the diffusive regime the van Hove function is vanishingly small for distances where $g(r)$ varies appreciably. Thus, we may replace $g(r)$ by its large- $r$ limit. This limit is 0 for the polymer pair-distribution function ${ }^{35}$ so that $\left\langle s_{\text {seg }}\right\rangle=1$, whereas it is 1 in the case of the melt. Furthermore, since $G_{s}$ is a normalized Gaussian, the integral over $\mathbf{r}_{i j}$ just gives 1 . Thus, we find $P_{\delta}=(\rho / M)\left(4 \pi \delta^{3} / 3\right)$. Together with Eq. (B1) this yields

$$
\langle s\rangle=1+\frac{4 \pi(0.065 M-1) \delta^{3}}{3 M} \rho .
$$

Using $\delta=0.55$ and $M=1020, \rho=0.9058$ at $T=1$ as well as $M=1200, \rho=1.0378$ at $T=0.46$ we find $\langle s\rangle=1.040$ and $\langle s\rangle=1.046$ at $T=1$ and 0.46 , respectively. These estimates are in good agreement with the simulation data of Fig. 7 at large times.

\section{APPENDIX C: CHARACTERISTIC TIMES}

Quantities such as $\alpha_{2}(t)$ or $N_{c, m}(t)$, exhibit a maximum at some time in the window of the $\alpha$-process. Ideal mode coupling theory predicts that, in principle, any time in the $\alpha$-regime may be chosen as a characteristic relaxation time of the $\alpha$-process (see, e.g., Refs. 2 and 56). That is, the theory suggests that the relaxation times $\tau_{X}$ of different quantities " $X$ " should only differ in prefactors, but scale with temperature in the same way

$$
\tau_{X}=\tau_{X}^{0}\left(\frac{T-T_{\mathrm{MCT}}}{T_{\mathrm{MCT}}}\right)^{-\gamma}\left(T \rightarrow T_{\mathrm{MCT}}^{+}\right) .
$$

Provided that $T$ is sufficiently close to $T_{\mathrm{MCT}}$, the exponent $\gamma$ is predicted to be independent of $X$. Contrary to that, the

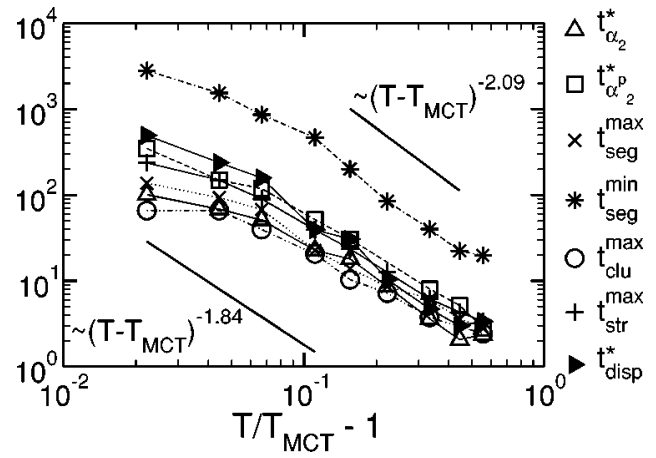

FIG. 18. $\log -\log$ plot of characteristic time scales vs $T / T_{\mathrm{MCT}}-1\left(T_{\mathrm{MCT}}\right.$ $=0.45)$. The times are: $t_{\alpha_{2}}^{*}\left(\right.$ maximum of $\left.\alpha_{2}\right), t_{\alpha_{2}^{p}}^{*}\left(\operatorname{maximum}\right.$ of $\left.\alpha_{2}^{p}\right), t_{\mathrm{seg}}^{\max }$ (maximum of $N_{c, m}$ ), $t_{\mathrm{seg}}^{\mathrm{min}}$ (minimum of $N_{c, m}$ ), $t_{\mathrm{clu}}^{\max }$ (maximum of the cluster size of mobile monomers; taken from Ref. 25), $t_{\mathrm{str}}^{\max }$ (maximum of $\langle s\rangle$, see Fig. 7), and $t_{\text {disp }}^{*}$ (a time where displacement-displacement correlation function is most pronounced; taken from Ref. 31). The solid lines labeled by the power law (C1) indicate the temperature dependence of the $\alpha$-relaxation time resulting from a quantitative MCT analysis [ $\gamma=2.09$ (Refs. 34 and 35)] and of the inverse diffusion coefficient $[\gamma=1.84$ (Refs. 35 and 38)].

range of validity of Eq. (C1), i.e., the highest temperature up to which the power law is observable, depends on $X .^{51,68}$

Fits to simulation data often show that Eq. (C1) only holds approximately. ${ }^{41,69}$ Very close to $T_{\mathrm{MCT}}$ deviations occur in (almost) all systems (colloidal suspensions appear to be an exception $\left.{ }^{16,17,20}\right)$. These deviations may be rationalized in the framework of MCT by relaxation mechanisms other than the cage effect that are not incorporated in the idealized theory. ${ }^{70,71}$ In the remaining temperature interval where Eq. (C1) can be applied, the fits often find the same result for $T_{\mathrm{MCT}}$, whereas $\gamma$ can depend on $X$. For the present model, for instance, the relaxation times derived from intermediate scattering functions yield a $\gamma$ that decreases with the modulus of the wave vector $\mathbf{q}$ and approaches $\gamma_{D}$, the exponent of the diffusion coefficient, in the low- $q$ limit. ${ }^{36,37}$ Since quantities like the mean-square displacements or the nonGaussian parameters are related to the small- $q$ behavior of the intermediate scattering functions, ${ }^{39,51}$ we expect that relaxation times derived from these quantities exhibit a temperature dependence that is compatible with $\gamma_{D}$, rather than with the exponent found at the maximum of the static structure factor.

Figure 18 shows the temperature dependence of various time scales introduced in this study, including also the time $t_{\text {clu }}^{\max }$ of Ref. 25 where the cluster size of mobile monomers is maximum. As expected, deviations from the power law (C1) are found for $T$ close to $T_{\mathrm{MCT}}$ (i.e., $T-T_{\mathrm{MCT}} \lesssim 0.02$ ). For larger temperatures the relaxation times roughly agree with Eq. (C1). Within the (admittedly large) statistical uncertainties of the data and perhaps with the exception of $t_{\text {clu }}^{\max }$ which seems to show a weaker increase ${ }^{25}$ the exponents are closer to $\gamma_{D}=1.84 \pm 0.02^{36}$ than to the result obtained at the maximum of the static structure factor $\left(\gamma \approx 2.09^{34,35}\right)$, as suggested above.

${ }^{1}$ J. Jäckle, Rep. Prog. Phys. 49, 171 (1986).

${ }^{2}$ W. Götze, in Proceedings of the Les Houches Summer School of Theoretical Physics, Les Houches 1989, Session LI, edited by J. P. Hansen, D. 
Levesque, and J. Zinn-Justin (North-Holland, Amsterdam, 1991), pp. 287-503.

${ }^{3}$ P. Lunkenheimer, U. Schneider, R. Brand, and A. Loidl, Contemp. Phys. 41, 15 (2000).

${ }^{4}$ H. Sillescu, J. Non-Cryst. Solids 243, 81 (1999).

${ }^{5}$ M. D. Ediger, Annu. Rev. Phys. Chem. 51, 99 (2000).

${ }^{6}$ R. Richert, J. Phys.: Condens. Matter 14, R703 (2002)

${ }^{7}$ S. C. Glotzer, J. Non-Cryst. Solids 274, 342 (2000).

${ }^{8}$ K. Schmidt-Rohr and H. W. Spiess, Phys. Rev. Lett. 66, 1991 (1991).

${ }^{9}$ U. Tracht, M. Wilhelm, A. Heuer, H. Feng, K. Schmidt-Rohr, and H. W. Spiess, Phys. Rev. Lett. 81, 2727 (1998).

${ }^{10}$ M. T. Cicerone and M. Ediger, J. Chem. Phys. 103, 5684 (1995).

${ }^{11}$ B. Schiener, R. Böhmer, A. Loidl, and R. V. Chamberlin, Science 274, 752 (1996).

${ }^{12}$ M. Yang and R. Richert, J. Chem. Phys. 115, 2676 (2001).

${ }^{13}$ Information about the length scale of this correlation can be directly measured via multidimensional NMR experiments. Typically, it is of the order of $3 \mathrm{~nm}$ close to $T_{g}$ (Ref. 9).

${ }^{14}$ E. Weeks, J. C. Crocker, A. C. Levitt, A. Schofield, and D. A. Weitz, Science 287, 627 (2000).

${ }^{15}$ W. K. Kegel and A. van Blaaderen, Science 287, 290 (2000).

${ }^{16}$ E. Bartsch, Curr. Opin. Colloid Interface Sci. 3, 577 (1998).

${ }^{17}$ W. van Megen, Transp. Theory Stat. Phys. 24, 1017 (1995).

${ }^{18}$ W. van Megen and S. M. Underwood, Phys. Rev. E 49, 4206 (1994).

${ }^{19}$ W. Götze and L. Sjögren, Phys. Rev. A 43, 5442 (1991).

${ }^{20}$ W. van Megen, T. C. Mortensen, J. Müller, and S. R. Williams, Phys. Rev. E 58, 6073 (1998)

${ }^{21}$ R. Yamamoto and A. Onuki, Phys. Rev. Lett. 81, 4915 (1998).

${ }^{22}$ W. Kob, C. Donati, S. J. Plimpton, P. H. Poole, and S. C. Glotzer, Phys. Rev. Lett. 79, 2827 (1997).

${ }^{23}$ C. Donati, J. F. Douglas, W. Kob, S. J. Plimpton, P. H. Poole, and S. C. Glotzer, Phys. Rev. Lett. 80, 2338 (1998).

${ }^{24}$ C. Donati, S. C. Glotzer, P. H. Poole, W. Kob, and S. J. Plimpton, Phys. Rev. E 60, 3107 (1999).

${ }^{25}$ Y. Gebremichael, T. B. Schrøder, F. W. Starr, and S. C. Glotzer, Phys. Rev. E 64, 051503 (2001).

${ }^{26}$ N. Giovambattista, S. V. Buldyrev, F. W. Starr, and H. E. Stanley, Phys. Rev. Lett. 90, 085506 (2003).

${ }^{27}$ B. Doliwa and A. Heuer, Phys. Rev. Lett. 80, 4915 (1998).

${ }^{28}$ B. Doliwa and A. Heuer, Phys. Rev. E 61, 6898 (2000).

${ }^{29}$ B. Doliwa and A. Heuer, J. Non-Cryst. Solids 307-310, 32 (2002).

${ }^{30}$ K. Vollmayr-Lee, W. Kob, K. Binder, and A. Zippelius, J. Chem. Phys. 116, 5158 (2002).

${ }^{31}$ C. Bennemann, C. Donati, J. Baschnagel, and S. C. Glotzer, Nature (London) 399, 246 (1999).

${ }^{32}$ C. Bennemann, W. Paul, K. Binder, and B. Dünweg, Phys. Rev. E 57, 843 (1998).

${ }^{33}$ K. Binder, J. Baschnagel, and W. Paul, Prog. Polym. Sci. 28, 115 (2003).

${ }^{34}$ C. Bennemann, J. Baschnagel, and W. Paul, Eur. Phys. J. B 10, 323 (1999).

${ }^{35}$ M. Aichele and J. Baschnagel, Eur. Phys. J. E 5, 229 (2001).

${ }^{36} \mathrm{M}$. Aichele and J. Baschnagel, Eur. Phys. J. E 5, 245 (2001).

${ }^{37}$ C. Bennemann, W. Paul, J. Baschnagel, and K. Binder, J. Phys.: Condens. Matter 11, 2179 (1999).

${ }^{38}$ C. Bennemann, J. Baschnagel, W. Paul, and K. Binder, Comput. Theor. Polym. Sci. 9, 217 (1999).

${ }^{39}$ S.-H. Chong and M. Fuchs, Phys. Rev. Lett. 88, 185702 (2002).

${ }^{40}$ J. P. Hansen and I. R. McDonald, Theory of Simple Liquids (Academic, London, 1986).

${ }^{41}$ S. Kämmerer, W. Kob, and R. Schilling, Phys. Rev. E 58, 2131 (1998).

${ }^{42}$ W. Kob and H. C. Andersen, Phys. Rev. E 51, 4626 (1995).

${ }^{43}$ F. Sciortino, P. Gallo, P. Tartaglia, and S.-H. Chen, Phys. Rev. E 54, 6331 (1996).

${ }^{44}$ S. Mossa, R. Di Leonardo, G. Ruocco, and M. Sampoli, Phys. Rev. E 62, $612(2000)$

${ }^{45}$ J. Horbach, W. Kob, and K. Binder, Philos. Mag. B 77, 297 (1998).

${ }^{46}$ J. Colmenero, F. Alvarez, and A. Arbe, Phys. Rev. E 65, 041804 (2002).

${ }^{47}$ R. Zorn, Phys. Rev. B 55, 6249 (1997).

${ }^{48} \mathrm{M}$. Aichele et al. (unpublished).

${ }^{49}$ V. Krakoviack, J. P. Hansen, and A. A. Louis, Europhys. Lett. 58, 53 (2002).
${ }^{50}$ M. Guenza, Phys. Rev. Lett. 88, 025901 (2002).

${ }^{51}$ M. Fuchs, W. Götze, and M. R. Mayr, Phys. Rev. E 58, 3384 (1998).

${ }^{52}$ M. Doi and S. F. Edwards, The Theory of Polymer Dynamics (Oxford University Press, Oxford, 1986).

${ }^{53}$ K. Kremer and G. S. Grest, in Monte Carlo and Molecular Dynamics Simulations in Polymer Science, edited by K. Binder (Oxford University Press, New York, 1995), pp. 194-271.

${ }^{54}$ Suppose that $G_{s}(\mathbf{r}, t)=\left[3 / 2 \pi g_{0}(t)\right]^{3 / 2} \exp \left[-3 \mathbf{r}^{2} / 2 g_{0}(t)\right]$. The fraction of the $6.5 \%$ of the most mobile monomers can then be expressed as: 0.065 $=4 \pi \int_{r *}^{\infty} \mathrm{d} r r^{2} G_{s}(\mathbf{r}, t)=(2 / \sqrt{\pi}) \Gamma\left(3 / 2, \sqrt{u^{*}}\right), \quad$ where $\quad u=3 r^{2} / 2 g_{0} \quad$ and $\Gamma(\alpha, x)$ is the incomplete gamma function. This condition yields $\sqrt{u^{*}}$ $\simeq 3.614$. Similarly, we can write for the MSD of the mobile monomers $g_{0, m}(t)=(4 \pi / 0.065) \int_{r^{*}}^{\infty} \mathrm{d} r r^{4} G_{s}(\mathbf{r}, t)=(4 / 0.065 \sqrt{9 \pi}) g_{0}(t) \Gamma\left(5 / 2, \sqrt{u^{*}}\right)$.

Using the identity $\Gamma(\alpha+1, x)=\alpha \Gamma(\alpha, x)+x^{\alpha} e^{-x}$, we obtain Eq. (6).

${ }^{55}$ This finding is not unreasonable. The mean-quartic displacement is more sensitive to large displacements than $g_{0}$. Thus, it particularly samples the large- $r$ wing of $G_{s}(\mathbf{r}, t)$ similar to $g_{0, m}$

${ }^{56}$ W. Götze, J. Phys.: Condens. Matter 11, A1 (1999).

${ }^{57}$ We have not considered the possibility of loops which would occur if a set of particles replaced each other in a circular fashion. In our analysis loops occurred very seldomly. Mostly, they were of length two (or four) and consisted of contiguous chain segments of length two. This means that bonded, neighboring monomers switch places. Because the loops were so rare, we decided not to analyze the results for open and closed strings separately. We note that in the LJ system studied in Ref. 23, longer loops were found.

${ }^{58}$ G. Strobl, The Physics of Polymers: Concepts for Understanding their Structures and Behavior (Springer, Berlin-Heidelberg, 1997).

${ }^{59}$ Note that we include "strings" of size one in analogy with percolation theory, where clusters of size one are customarily included in the evaluation of mean cluster size, and with equilibrium polymerization, where polymers of size one are customarily included in the calculation of mean polymer size.

${ }^{60}$ Y. Gebremichael, M. Vogel, and S. C. Glotzer (unpublished).

${ }^{61}$ R. Bellissent, L. Descotes, and P. Pfeuty, J. Phys.: Condens. Matter 6, A211 (1994)

${ }^{62}$ Y. Rouault and A. Milchev, Phys. Rev. E 51, 5905 (1995).

${ }^{63}$ S. C. Greer, Adv. Chem. Phys. 94, 261 (1996).

${ }^{64}$ J. P. Wittmer, A. Milchev, and M. E. Cates, J. Chem. Phys. 109, 834 (1998).

${ }^{65}$ Suppose we have a vector $\mathbf{d}$ on the unit sphere $S^{2}$ connecting the origin to a point on the surface of $S^{2}$ in direction $(\theta, \phi) . \theta \in[0, \pi[$ is the latitude and $\phi \in[0,2 \pi[$ the longitude. Furthermore, let $\hat{\mathbf{n}}$ be the vector from the origin to the north pole $(\theta=0, \phi=0)$. Then, the angles defined in Eqs. (16) and (17) correspond to a measurement of the latitude $\theta$, the angle between $\mathbf{d}$ and $\hat{\mathbf{n}}$, after integration over $\phi$. Because the sector of the unit sphere for fixed $\theta$ is small if $\theta$ is close to the poles, but large if it is close to the equator, the $\phi$-integrated probability of an isotropic distribution of vectors on $S^{2}, P_{\text {iso }}(\theta)$, is maximum at $\theta=90^{\circ}$

${ }^{66}$ The symmetry in Fig. 13 arises because we calculate $P_{d, r}\left(\theta\left[\mathbf{d}_{i}(t), \mathbf{r}_{i j}\right]\right)$ by averaging over the displacements $\mathbf{d}_{i}(t)$ and $\mathbf{d}_{j}(t)$ for the same $\mathbf{r}_{i j}$. Thus, the rise of $P_{d, r}(\theta) / P_{\text {iso }}(\theta)$ close to $\theta=0^{\circ}$ results from motions of particle $i$ in direction of $\mathbf{r}_{i j}$, whereas that close to $\theta=180^{\circ}$ comes from displacements of particle $j$ against the orientation of $\mathbf{r}_{i j}$. Note that the analysis of Ref. 23 only consider the former case. Therefore, the probability distribution is asymmetric (see Fig. 3 of Ref. 23).

${ }^{67}$ An analysis of $P_{d, d}\left(\theta\left[\mathbf{d}_{i}(t), \mathbf{d}_{j}(t)\right]\right) / P_{\text {iso }}\left(\theta\left[\mathbf{d}_{i}(t), \mathbf{d}_{j}(t)\right]\right)$ in the time interval $1 \leqq t \leqslant 10^{4}$ shows that $P_{d, d}\left(\theta\left[\mathbf{d}_{i}(t), \mathbf{d}_{j}(t)\right]\right)>P_{\text {iso }}\left(\theta\left[\mathbf{d}_{i}(t), \mathbf{d}_{j}(t)\right]\right)$ for $\theta \lesssim 60^{\circ}$, whereas $P_{d, d}\left(\theta\left[\mathbf{d}_{i}(t), \mathbf{d}_{j}(t)\right]\right) \rightarrow 0$, as $\theta \rightarrow 180^{\circ}$.

${ }^{68}$ T. Franosch, M. Fuchs, W. Götze, M. R. Mayr, and A. P. Singh, Phys. Rev. E 55, 7153 (1997).

${ }^{69}$ W. Kob and H. C. Andersen, Phys. Rev. E 52, 4134 (1995).

${ }^{70}$ M. Fuchs, W. Götze, S. Hildebrand, and A. Latz, J. Phys.: Condens. Matter 4, 7709 (1992).

${ }^{71}$ W. Götze and L. Sjögren, Z. Phys. B: Condens. Matter 65, 415 (1987). 